\title{
A systematic molecular simulation study of ionic liquid surfaces using intrinsic analysis methods
}

\author{
György Hantal ${ }^{1,2}$, Iuliia Voroshylova ${ }^{2}$, M. Natália D S Cordeiro ${ }^{2}$ and Miguel \\ Jorge $\mathrm{e}^{1, *}$ \\ ${ }^{1}$ LSRE - Laboratory of Separation and Reaction Engineering - Associate Laboratory LSRE/LCM, \\ Faculdade de Engenharia, Universidade do Porto, Rua Dr. Roberto Frias, 4200-465 Porto, Portugal \\ ${ }^{2}$ REQUIMTE - Associate Laboratory, Faculdade de Ciências, Universidade do Porto, Rua do Campo \\ Alegre, 687, 4169-007 Porto, Portugal
}

\begin{abstract}
In this paper, we apply novel intrinsic analysis methods, coupled with bivariate orientation analysis, to obtain a detailed picture of the molecular-level structure of ionic liquid surfaces. We observe pronounced layering at the interface, alternating non-polar with ionic regions. The outermost regions of the surface are populated by alkyl chains, which are followed by a dense and tightly packed layer formed of oppositely charged ionic moieties. We then systematically change the cation chain length, the anion size, the temperature and the molecular model, to examine the effect of each of these parameters on the interfacial structure. Increasing cation chain length promotes orientations in which the chain is pointing into the vapor, thus increasing the coverage of the surface with alkyl groups. Larger anions promote a disruption of the dense ionic layer, increasing the orientational freedom of cations and increasing the amount of free space. The temperature had a relatively small effect on the surface structure, while the effect of the choice of molecular model was clearly significant, particularly on the orientational preferences at the interface. Our study demonstrates the usefulness of molecular simulation methods in the design of ionic liquids to suit particular applications.
\end{abstract}




\section{Introduction}

Ionic liquids (ILS) constitute an extremely promising new class of materials, with the possibility to revolutionize chemical technologies in the XXI ${ }^{\text {st }}$ century. Their almost negligible vapor pressure together with their broad liquidus range and large thermal stability make them almost universally applicable as green solvents,[1] replacing traditional volatile organic compounds in chemical synthesis,[2] separation,[3] gas capture processes, [4] solar cells,[5] and catalysis.[6] They also display a wide electrochemical window, which enhances their significance for usage in electrochemical devices. [7] The huge number of possible ILs that can, a priori, be synthesized offers the possibility for task-specific tailoring of IL properties by finding the most appropriate combinations of cations and anions.[8] This fact in particular significantly increases their potential and expands the range of possible applications.

Although most experimental and theoretical studies have been devoted to the understanding of bulk-phase IL properties, a large number of practical applications depend on an in-depth knowledge of IL interfacial properties - important examples are liquid extraction or catalysis. Interfaces, however, are much more difficult to probe experimentally, most often requiring advanced techniques,[9-12] which have only recently started to become available. On account of the complexity of the system, different experimental methods used to analyze the IL surface have sometimes led to seemingly conflicting interpretations.[13] In this context, molecular simulation methods present a valuable complement to experiments, enabling one to probe molecular properties in detail, going even beyond the experimental resolution.[14]

It is thus not surprising that several molecular simulation studies have already been dedicated to elucidating the interfacial characteristics of ILs.[15-23] In the vast majority of these studies, the interface is considered as a static entity that is analyzed relative to a fixed reference plane, thus yielding spatially averaged properties that are smoothed by thermal fluctuations of the interface position (what we call the "global view" of the interface). This "global" (i.e. non-intrinsic) view of the surface restrains the scope of the possible information that can be gained from such analyses since at the molecular scale, the position and the shape of fluid interfaces continuously change in time. The superposition of thermally activated capillary waves onto the inherent corrugation of the interface causes the intrinsic surface structure to remain hidden from conventional molecular simulations.[24] Recent years have seen the advent and consolidation of intrinsic analysis methods that are able to circumvent the limitations described above.[25-28] These novel techniques make it possible to decouple capillary waves from the intrinsic roughness of the interface and to unequivocally separate true interfacial molecules from bulk molecules at any moment of the simulation. This makes it also possible to analyze interfacial properties in terms of the intrinsic distance from the instantaneous interface. Note that the application of an intrinsic analysis technique is particularly desirable when comparing simulation results to experiments that probe only interfacial molecules. Figure 1 illustrates the basic differences between global and intrinsic views of simulated interfacial systems. 


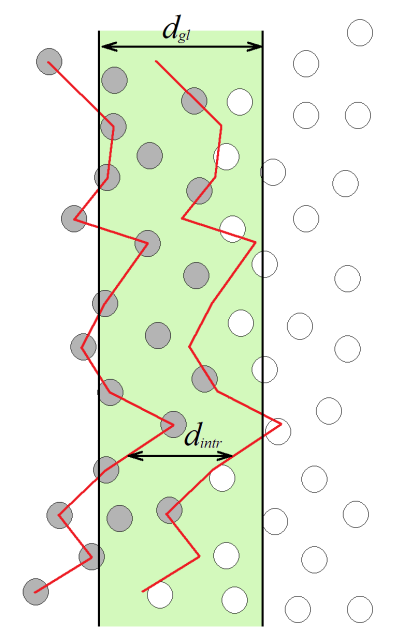

Figure 1 - Diagram depicting the global and intrinsic views of a fluid interface. The black lines enclose the light green slab of width $d_{g l}$ used to compute properties in the global framework, while the red lines enclose an intrinsic slab of width $d_{i n t r}$. The intrinsic slab contains contributions from atoms (in gray) that are all not farther than $d_{\text {intr }}$ from the corrugated surface. The global slab, on the contrary, includes contributions from atoms that are at significantly different distances from the surface, thus artificially smoothing the computed profiles.

In our previous study we applied intrinsic analysis methods to an IL surface (the room-temperature liquid/vapor interface of 1- $n$-butyl-3-methylimidazolium hexafluorophosphate, BMIMPF 6 ) for the first time, demonstrating the broad spectrum of information one can obtain by using these techniques.[22] We compared the intrinsic and global density profiles of the whole system, as well as number density profiles of individual atoms. By combining these profiles with bivariate orientation analyses, we were able to identify the three most prominent orientations of the imidazolium rings of the cations and provide an estimate for their relative prevalence. This picture was completed by a determination of the orientational preferences of the alkyl chains. This detailed study revealed that in the majority of cases the imidazolium ring is perpendicular to the interface with the butyl chain protruding into the vapor. In the second preferred orientation, the rings lay parallel to the interface and the butyl chains again protrude into the vapor. A small but nonnegligible amount of molecules, however, displayed a hitherto unidentified orientational preference - the ring was perpendicular to the interface but this time the methyl group pointed to the vapor whereas the alkyl chains were on the liquid side of the interface.

The aim of the present paper is to extend our study to a broad range of IL systems and temperatures using our intrinsic analysis method. $[27,28]$ We focus once again on the imidazolium family since it is one of the most widely employed classes of ILs. We are particularly interested in studying the effect of changing the cation chain length and the size of the anion on the structure of the IL liquid/vapor interface. We also wish to understand the underlying basic interactions and their relative importance in determining the interfacial structure. For this reason, several simulations were carried out by systematically changing one property while leaving the others unchanged. The BMIMPF 6 
system at $360 \mathrm{~K}$ was chosen as a reference system. Three different anions of different size $\left(\mathrm{Cl}^{-}, \mathrm{BF}_{4}{ }^{-}\right.$ and $\mathrm{PF}_{6}{ }^{-}$) as well as three different alkyl chain lengths (ethyl (EMIM), butyl (BMIM) and hexyl (HMIM) - see Figure 2) were studied. Furthermore, we tested the effect of temperature $(298 \mathrm{~K}, 330 \mathrm{~K}$ and $360 \mathrm{~K}$ ) and of using different molecular models. The conditions of all the simulations are summarized in Table 1.

\section{EMIM $^{+}$}
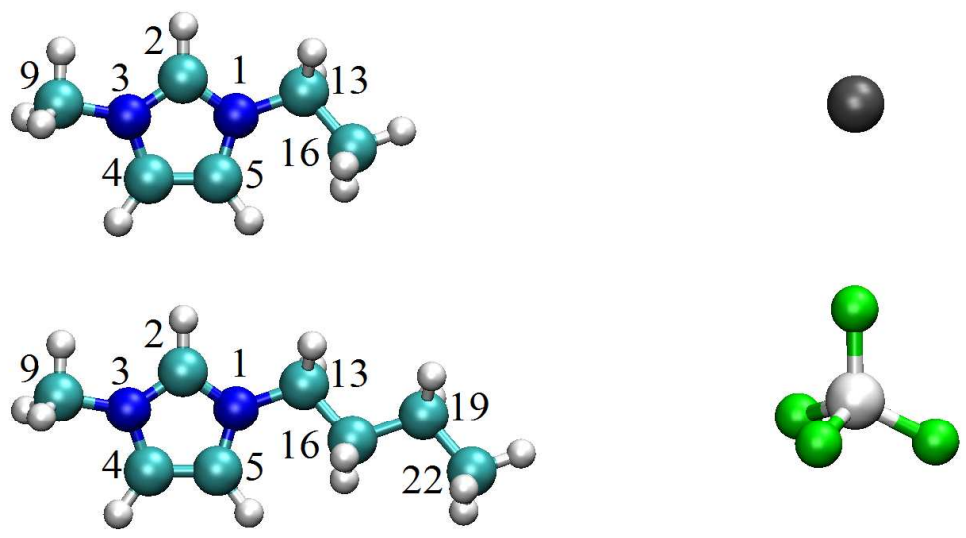

$\mathrm{BF}_{4}^{-}$

$\mathrm{HMIM}^{+}$
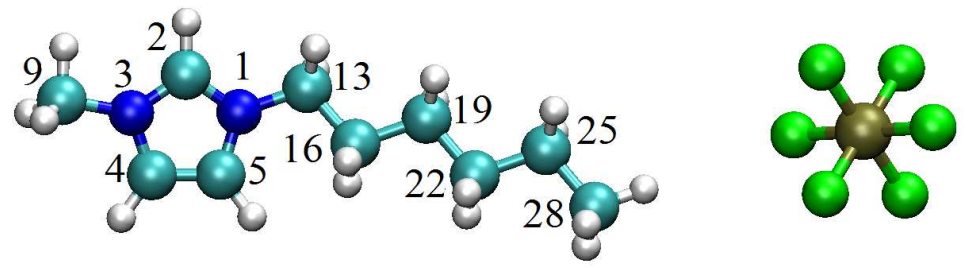

$\mathrm{PF}_{6}^{-}$

Figure 2 - Diagram of the ionic liquids studied in this paper (cations on the left and anions on the right). The numbering for non-hydrogen atoms of the cations is also shown.

Table 1 - Details of all systems studied in this paper, indicating those used as reference systems. In each row, the characteristics that are varied compared to Reference I are marked in bold font. AA stands for an all-atom model, while UA stands for a united atom representation of the alkyl groups. In the last column, the total charge on each ion is also given.

\begin{tabular}{|c|c|c|c|c|c|}
\hline & Cation & Anion & Temperature & Force field & Remarks \\
\hline \multirow[t]{2}{*}{ Reference II $^{1}$} & $\mathrm{BMIM}^{+}$ & $\mathrm{PF}_{6}^{-}$ & 298.15 K & CLaP & AA, \pm 1.0 \\
\hline & $\mathrm{BMIM}^{+}$ & $\mathrm{PF}_{6}^{-}$ & $330.15 \mathrm{~K}$ & CLaP & AA, \pm 1.0 \\
\hline \multirow[t]{5}{*}{ Reference I } & $\mathrm{BMIM}^{+}$ & $\mathrm{PF}_{6}^{-}$ & $360.15 \mathrm{~K}$ & CLaP & $\mathrm{AA}, \pm 1.0$ \\
\hline & $\mathrm{BMIM}^{+}$ & $\mathrm{BF}_{4}^{-}$ & $360.15 \mathrm{~K}$ & CLaP & $\mathrm{AA}, \pm 1.0$ \\
\hline & $\mathrm{BMIM}^{+}$ & $\mathrm{Cl}^{-}$ & $360.15 \mathrm{~K}$ & CLaP & $\mathrm{AA}, \pm 1.0$ \\
\hline & EMIM $^{+}$ & $\mathrm{PF}_{6}^{-}$ & $360.15 \mathrm{~K}$ & CLaP & $\mathrm{AA}, \pm 1.0$ \\
\hline & HMIM $^{+}$ & $\mathrm{PF}_{6}^{-}$ & $360.15 \mathrm{~K}$ & CLaP & $\mathrm{AA}, \pm 1.0$ \\
\hline
\end{tabular}




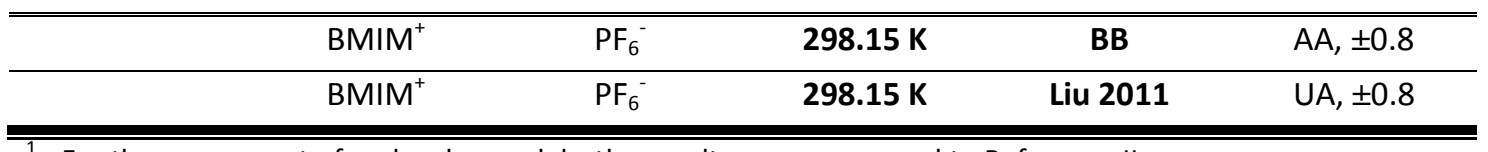

${ }^{1}$ - For the assessment of molecular models, the results were compared to Reference II.

\section{II computational details}

\section{II.1 Simulations}

Canonical ensemble molecular dynamics simulations were performed with the Gromacs program package.[29] Each system contained an IL slab with a thickness of 6-8 nm comprising 512 ion pairs. Details of the $\mathrm{BMIMPF}_{6}$ simulation at $298 \mathrm{~K}$ were given in our previous publication.[22] For the remaining systems described in Table 1, the length of the simulation box parallel to the interface was fixed at $5 \mathrm{~nm}$, whereas in the surface normal direction the length was fixed to $16 \mathrm{~nm}$. The simulations were carried out at constant temperatures using the velocity rescaling method.[30] Electrostatic interactions were truncated in real space beyond a cut-off radius of $1.5 \mathrm{~nm}$. To account for the long range part of the electrostatic interaction the PME method was used.[31] Dispersion interactions were truncated by smoothly decreasing to zero between 1.1 and $1.2 \mathrm{~nm}$, with long range corrections applied beyond this distance. All systems were equilibrated for $12 \mathrm{~ns}$, except for $\mathrm{BMIMCl}$ at $360 \mathrm{~K}$. This system turned out to take much longer to equilibrate than any of the other systems. To improve the equilibration, we performed an extra simulation run at $450 \mathrm{~K}$ for $20 \mathrm{~ns}$ followed by a re-equilibration at $360 \mathrm{~K}$ for another $20 \mathrm{~ns}$. Even with this procedure, we were not completely certain that the $\mathrm{BMIMCl}$ was indeed equilibrated. The implications of this are discussed further in the results section.

After equilibration, a $40 \mathrm{~ns}$ long production was carried out. The time step of the simulations was 2 fs. Configurations during the production were saved at every $2 \mathrm{ps}$, thus resulting in a sample of 20000 configurations. According to the original parameterization of the force field, all $\mathrm{C}-\mathrm{H}$ bonds were kept fixed using the SHAKE algorithm.[32] Flexible anions were used in all simulations, except for Reference II (Table 1) for which rigid anions were used. We performed several tests to assess the effect of anion flexibility on the interfacial structure, and observed this effect to be negligible.

Most of the simulations were performed with the widely used force field developed by CanongiaLopes and Padua (CLaP, see Table 1).[33] The reason for choosing this force field was mainly that it includes parameters for a large variety of anions and cations, which gives it wide applicability. The force field by Bhargava and Balasubramanian (BB) was also studied.[34] This is a refinement of the CLaP model for $\mathrm{BMIMPF}_{6}$ and is available only for this particular IL. The authors achieved good agreement with experiment for static and dynamic properties by fine-tuning some of the LennardJones parameters as well as by resetting atomic charges to yield a total charge of \pm 0.8 on each ion (compared to \pm 1.0 in the CLaP model). The third force field we tested has been developed by Liu et al. (Liu 2011).[35] This very recent force field is based on the earlier broad all-atom force field by the same group [36] but includes two main changes compared to the old version: i) it uses united atoms 
for the methyl and methylene groups; ii) all charges are uniformly rescaled to yield a total charge of \pm 0.8 on each ion. This refined force field displayed far better dynamic properties than the original version.[35]

\section{II.2 Intrinsic analyzing method}

The technique we used here for calculating intrinsic properties of the interfaces is based on two steps: i) detecting the true set of atoms that are located precisely at the interface; ii) creating a mathematical surface that passes through these atoms, and computing properties relative to this surface. This is schematically depicted in Figure 1, where the interfacial atoms are represented by the left-most gray circles, connected by a red line that represents the mathematical surface. The basis of the method will be shortly recalled here, but the reader is referred to Refs $28,37,38$ for further details. For the first step, we employed the 'Identification of Truly Interfacial Molecules' method (ITIM).[28] First, a rectangular grid is constructed above the liquid phase to be probed. Then, in every single configuration of the sample, a series of probe spheres are dropped from each grid point onto the surface. Interfacial atoms are defined as the first atoms to come into contact with at least one of the falling probe spheres. Interfacial molecules are defined as those having at least one interfacial atom.

In the second step of our method, we generate consecutive corrugated slabs along the surface normal, at equal distance from each other, that match the real shape of the surface layer (see region between the two red lines in Figure 1). We are thus able to compute intrinsic profiles relative to the true position of the interface, which reveal the underlying structure of the fluid. This is in contrast to global analysis, in which the profiles are artificially smoothed by the use of rectangular slabs and a fixed reference frame (see green region in Figure 1). To build the mathematical surface defining the surface layer, a triangular interpolation algorithm was used. This was found to be the most efficient way of accurately computing intrinsic profiles based on a finite set of interfacial atomic positions.[38]

The ITIM method contains two free parameters: the grid spacing and the radius of the probe sphere. Provided the grid is fine enough, the results are not significantly affected by the grid spacing. It has been shown that a good choice for this parameter is $0.05 \mathrm{~nm}$.[37] As for the probe sphere radius, an optimal value can be found, at least for relatively simple molecular liquids, by systematically analyzing the effect of this parameter on the symmetry of the distribution of interfacial molecules.[38] Unfortunately, it is not clear whether this approach can be directly applied to complex systems like ionic liquids (with two molecular components, at least one of which has a strongly asymmetric shape). Nevertheless, a reasonable value of the probe sphere radius can be intuitively chosen - it should be close to the characteristic size of the atoms constituting the system [28] and should be smaller than the amplitude of the smallest capillary waves.[27] Choosing considerably larger probe spheres will result in an underestimation of the interfacial set of molecules, while probe spheres that are too small will detect molecules belonging to the second 
molecular layer beneath the interface, thus overestimating the set of interfacial molecules. Based on these considerations, we set the radius of the probe sphere to $0.2 \mathrm{~nm}$, as this value was already used successfully in several interfacial studies and was shown to provide sensible results for different molecular fluids.[39].

\section{Results and discussion}

\section{III.1 Reference System}

Before addressing the question of the effect of different parameters on the interfacial properties, a detailed analysis of our reference system - BMIMPF${ }_{6}$ at $360 \mathrm{~K}$ - will be presented in this section. This will also serve the purpose of exemplifying the properties that can be calculated using the intrinsic analysis, and how they can be used to obtain a detailed view of the surface structure. The changes in the interface structure caused by changing the molecular constituents of the IL and by changing the temperature will be analyzed in the following section.

\section{III.1.1 Density profiles}

Global and intrinsic density profiles were computed along the surface normal direction. Density profiles for all the systems studied were highly symmetric (except for the BMIMCl system, see discussion below), which is an indication that the simulations are well equilibrated. Therefore all profiles shown were averaged over the two sides of the liquid slab and were also shifted along the $z$ axis so that the center of mass of the system is at $z=0 \mathrm{~nm}$.

Figure 3 shows the global and intrinsic mass density profiles of the system as well as global and intrinsic number density profiles of individual atoms (see Figure 2 for atom nomenclature) as obtained in $\mathrm{BMIMPF}_{6}$ at $360 \mathrm{~K}$. 
GLOBAL PROFILES
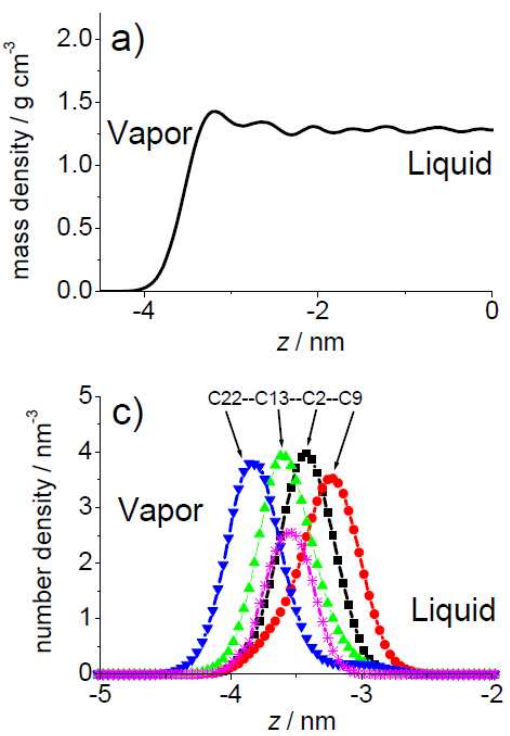

INTRINSIC PROFILES
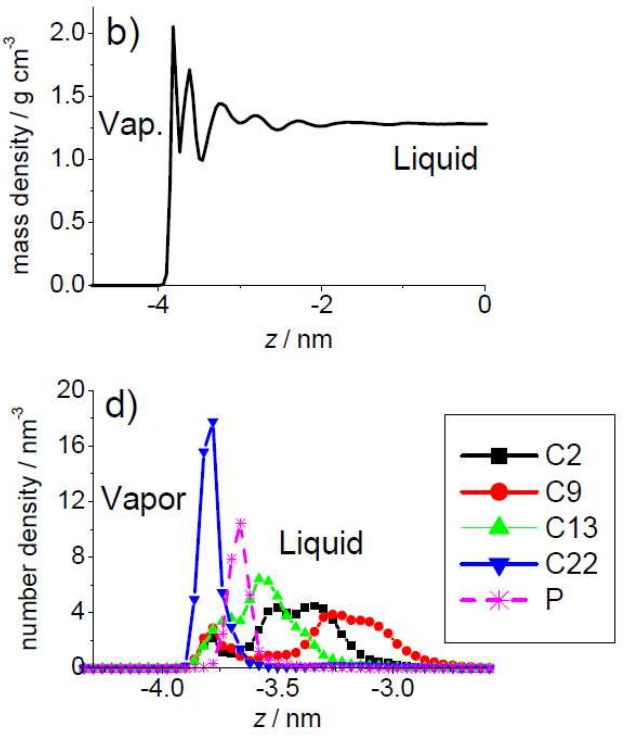

Figure 3 - Density profiles for $\mathrm{BMIMPF}_{6}$ at $360 \mathrm{~K}$ : a) global mass density profile; b) intrinsic mass density profile; c) global number density profiles for selected atoms that belong to interfacial molecules; d) intrinsic number density profiles for selected atoms that belong to interfacial molecules.

Panels a and $\mathrm{b}$ of Figure 3 show global and intrinsic mass density profiles, respectively. The global mass density shows a moderate density enhancement at the interface, followed by a mildly oscillating shape decaying toward the bulk. The intrinsic density exhibits, however, a sharp and high first peak located at the average location of the interface, which is entirely due to the interfacial atoms detected by the ITIM algorithm. This is followed by a second peak, which is attributed mostly to atoms that are not precisely at the surface but that are bound to interfacial atoms typically directly or through a few bonds. After these two peaks, there is a deep trough that separates them from a zone where atoms belonging to interfacial molecules and atoms belonging to the second molecular layer contribute to the density profile more or less to the same extent.[38] The fourth peak, however, is fully attributed to molecules residing beneath the interface. The profile exhibits a few more oscillations that eventually decay as one moves into the bulk liquid. It is also worth noticing that the unphysical density oscillations seen in the bulk region of the global profiles (for $z$ >$2 \mathrm{~nm}$ ), which have also been observed in most previous simulation studies of IL interfaces, are suppressed when the profiles are calculated in the intrinsic framework. Although more systematic studies are necessary to fully clarify this point, it suggests that presence of the ripples in the profile is related to an improper global description of the interface.

Number density profiles for the individual atoms that showed the most structured profiles: C2 (i.e. the ring carbon between the two nitrogens), $\mathrm{C9}$ (the carbon atom of the methyl group in position 3), C13 and C22 (i.e. the first and last carbon atoms in the butyl chain, respectively), as well as for the anion $\mathrm{P}$ atom, are shown in Figures $\mathrm{c}$ and $\mathrm{d}$ relative to the global and intrinsic reference frames, 
respectively. In panel c, the peaks of the interfacial atoms appear to follow each other in a consecutive order starting with the last butyl atom on the vapor side and ending with the methyl group in the liquid. This suggests that the cations are mainly oriented with their butyl chains toward the vapor phase. However, the asymmetric shape of the profiles anticipates that other orientations can also be expected. Indeed, the intrinsic density profiles shown in panel d display distinct density regions separated by marked minima and maxima. This high degree of structure in the atomic density profiles arises from the fact that the IL cations exhibit a variety of molecular orientations, as observed previously for the same system at room temperature.[22] It is also important to notice in the global profiles for the $P$ atom that the anion is located close to the position of the imidazolium ring atoms, with a slight shift toward the vapor side.

Using our intrinsic analysis method, one may also obtain information about the actual atoms that are located at the surface. The distribution of interfacial atoms for the reference system is shown in Figure 4. It is clear that most surface atoms belong to the alkyl chain of the cation. Nevertheless, it is important to notice a non-negligible amount of atoms belonging to the cation ring and even to the methyl substituent, as well as a significant percentage of atoms belonging to the anion. This shows that the anion is clearly "visible" from the vapor side, which agrees with experimental and other theoretical observations (see, e.g., Refs 11 and 20).

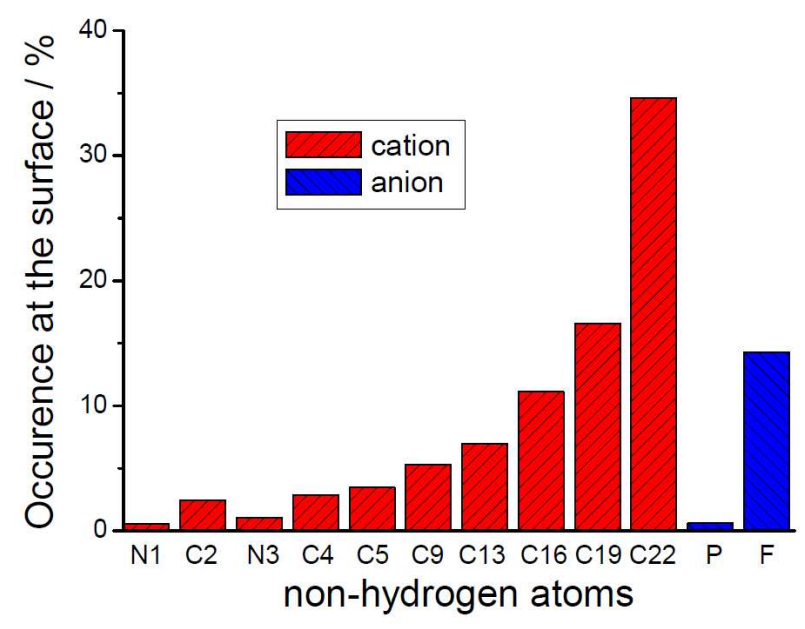

Figure 4 - Frequency of non-hydrogen atoms in $\mathrm{BMIMPF}_{6}$ at $360 \mathrm{~K}$ identified as interfacial by our intrinsic analysis method. Contributions from hydrogen atoms are attributed to the carbon atoms to which they are bonded. For atom numbering see Figure 2.

\section{III.1.2 Orientation analysis of the cations}

The density profiles discussed above clearly point to a distribution of orientations, rather than to a single molecular arrangement at the surface. To further investigate the orientation preferences of cations at the interface, we performed a bivariate orientation analysis by adapting the method of Jedlovszky et al.[40] In this method the orientation of a characteristic vector is described by its polar 
angular coordinates in a coordinate frame fixed to a rigid body. In our case the vector is the surface normal, while the coordinate frame is fixed to the imidazolium ring in the following way: the origin of the coordinate frame is located on the C2 atom; the $z$ axis goes along the symmetry axis of the ring and points from $\mathrm{C} 2$ toward the midpoint between atoms $\mathrm{C} 4$ and $\mathrm{C} 5$; the $\mathrm{y}$ axis is parallel to the vector pointing from N3 to N1; finally, the $x$ axis is perpendicular to the $x y$-plane. The definition of the coordinate frame is illustrated in Figure 5.

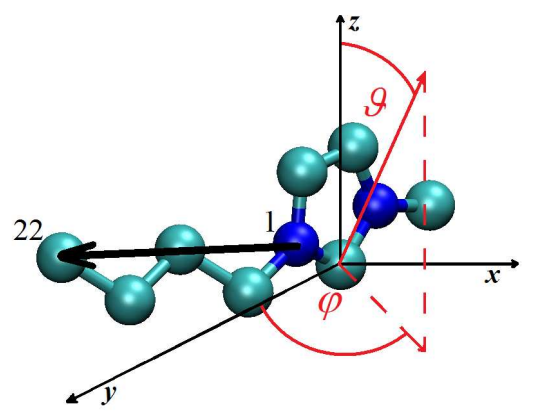

Figure 5 - Diagram showing the coordinate axes used for the bivariate orientation analysis of the preferred orientations of cation rings. The black vector pointing from N1 to C22 is used to characterize chain orientations (see text for details).

The obtained bivariate orientation distributions can be plotted as two-dimensional maps using brighter tones to represent higher probabilities, thus giving us a complete description of the ring orientations. If we plot the complete orientation profile for the surface layer, it is dominated by a large peak corresponding to the ring oriented perpendicular to the interface with the butyl chain pointing to the vapor (see Figure S1 in ref. 22). To identify the full range of preferred orientations, one must decouple the orientation map using the intrinsic density profiles for individual atoms, shown I Figure 3d. The orientation maps in different intrinsic density regions for atoms C2, C9 and C22 together with the individual density profiles are plotted in Figure 6. Similarly to what we observed in our previous study,[22] three main orientations can be identified. The peak at $\cos \theta=-$ $0.17\left(\theta=100^{\circ}\right)$ and $\varphi=0^{\circ}$, denoted as orientation $\mathrm{I}$, is the most dominant peak, and corresponds to an arrangement where the imidazolium ring is perpendicular to the interface and the butyl chain is on the vapor side, but the ring is slightly tilted in such a way that the C2-C5 vector is almost perpendicular to the surface normal. Another peak emerges at $\cos \theta=-0.04\left(\theta=92^{\circ}\right)$ and $\varphi=86^{\circ}$, which corresponds to an orientation where the imidazolium rings are lying parallel to the interface (orientation II). This peak presents large broadness along $\cos \theta$, extending between values of around -0.6 and 0.6. This means that the ring has a large freedom to nod along the NN axis, with this axis remaining perpendicular to the surface normal. A third orientation, denoted as orientation III, can also be identified at $\cos \theta=-0.12\left(\theta=97^{\circ}\right)$ and $\varphi=180^{\circ}$, representing an orientation in which the ring is once again perpendicular to the interface but now the methyl group is on the vapor side of the interface, whilst the butyl chain is on the liquid side. The three main orientations of the IL cation are depicted in Figure 7. 

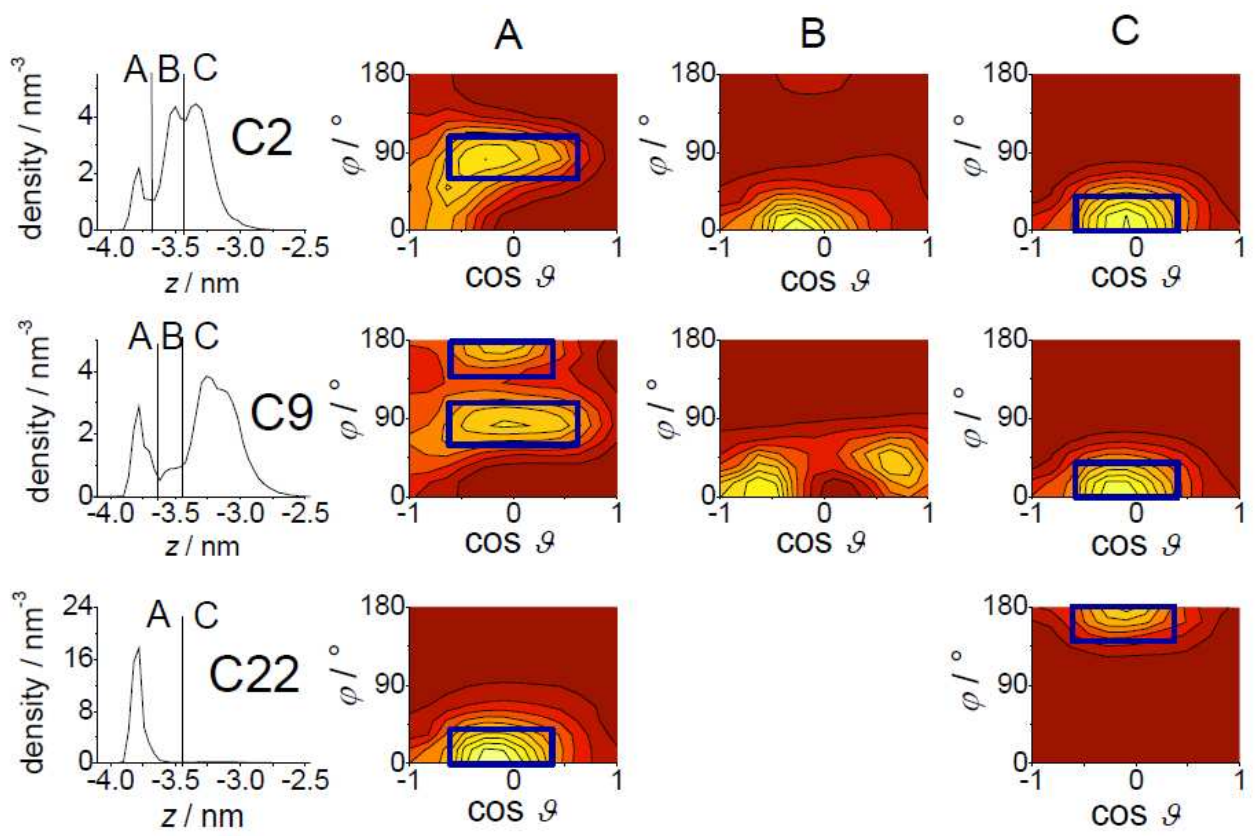

Figure 6 - Two-dimensional orientation maps for the cation rings in $\mathrm{BMIMPF}_{6}$ at $360 \mathrm{~K}$. Ligther (yellow) tones denote higher probabilities, while darker (red) tones mean lower probabilities. Each map corresponds to a distinct region in the intrinsic density profile of a selected atom of the cation. Results are shown for $\mathrm{C} 2, \mathrm{C} 9$ and $\mathrm{C} 22$ atoms, with the respective density profiles presented on the left to show the regions that correspond to each orientation map. Blue rectangles superimposed onto the maps delimit the orientation zones for each preferential ring orientation (see text for details).

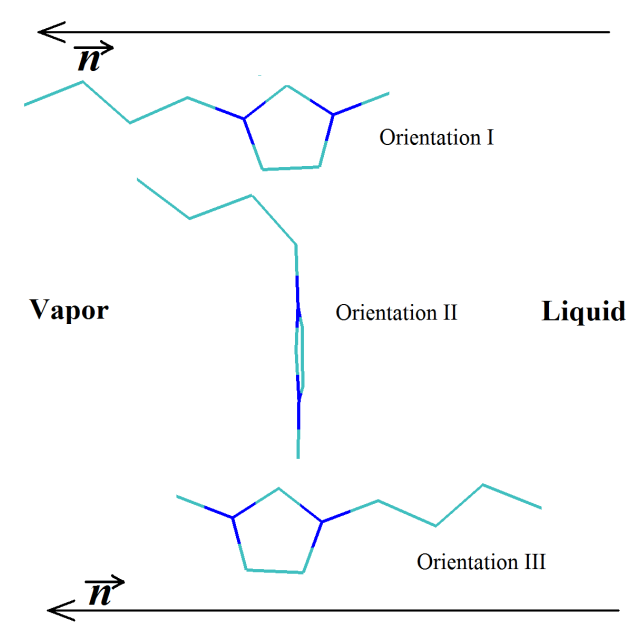

Figure 7 - Diagram representing the three preferred cation orientations in the imidazolium family of ionic liquids. Nitrogen atoms are represented in dark blue and carbon atoms in cyan.

We have calculated orientation maps analogous to those in Figure 6 for all systems described in Table 1 . In all cases, the maps were qualitatively similar, indicating always the same three typical 
orientations. Therefore, we do not present the full maps in section III.2, but instead adopt a different approach, described in the following sub-section, to quantify the change in orientation preferences as we change the simulation conditions.

\section{III.1.3 Prevalence of the different ring orientations}

In our previous study on $\mathrm{BMIMPF}_{6}$ at $298 \mathrm{~K}$ we estimated the fraction of cations in each different orientation by simply integrating the density regions corresponding to a given prominent orientation.[22] Specifically, we integrated zone C of C22 atoms to get the frequency of orientation III. If one integrates zone A of C9 atoms, that yields an estimate for the prevalence of orientations II and III together, thus one should subtract the prevalence for orientation III (obtained previously) to get an estimate for orientation II alone. An estimate for the prevalence of orientation I was obtained by integrating zone $\mathrm{C}$ for $\mathrm{C}$. This analysis allowed us to conclude that orientation I was by far the most predominant, followed by orientations II and III, in that order.[22]

Although the integration of distinct density regions can provide a rough estimate in absolute terms for the ratio of the different orientations of the cation rings to the total number of cations, the separation of the density regions is rather arbitrary and may depend on the precise shape of the profiles. This makes it difficult to be systematic when comparing values obtained for the different IL systems to identify any trends arising from changing the IL properties. This led us to implement here a somewhat different approach. We define zones on the orientation maps for each different preferred arrangement, and consider molecules to have a given orientation only if they fall into the corresponding zone. This classification will not provide absolute values for the ratio of the different molecular orientations because it will account for the corresponding molecules only partially. However, because the orientation zones are always defined in the same way for all the different systems, one is able to draw conclusions and establish trends when comparing them to each other. To this end, we defined the following zones: orientation I: $-0.6<\cos \theta<0.4,0^{\circ}<\varphi<40^{\circ}$, orientation II: $-0.6<\cos \theta<0.6,60^{\circ}<\varphi<110^{\circ}$ and orientation III: $-0.6<\cos \theta<0.4,140^{\circ}<\varphi<180^{\circ}$. These limits are marked as blue rectangles in Figure 6 . 


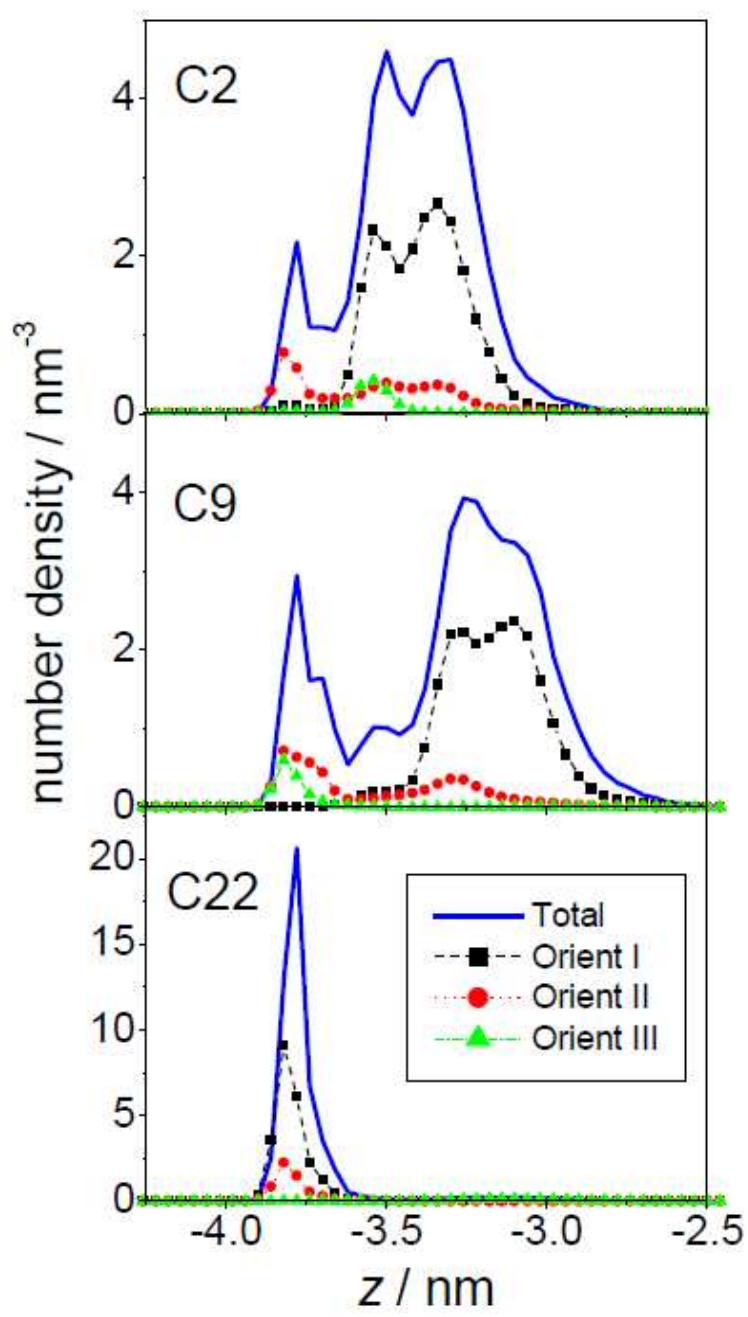

Figure 8 - Intrinsic density profiles for selected atoms belonging to interfacial molecules, split into contributions arising from each preferential ring orientation, as given by the blue rectangles in

Figure 6.

Figure 8 represents the partial intrinsic density profiles of the $\mathrm{C} 2, \mathrm{C} 9$ and $\mathrm{C} 22$ atoms corresponding to the different orientation zones defined above. This picture reveals that although a given orientation may dominate certain density regions, this does not necessarily exclude its occurrence in other zones, dominated by different orientations. For example, molecules with orientation II are dominant on the left-most peaks of the C2 and C9 distributions, but can also be found in deeper regions of these density profiles. For $\mathrm{BMIMPF}_{6}$ at $360 \mathrm{~K}$, integration of the orientation profiles within each zone yields $45.0 \%, 11.2 \%$ and $3.0 \%$ for orientations I, II and III, respectively. Note that this analysis allows us to classify only about $60 \%$ of the total number of cations - the remainder are in orientations that either deviate slightly from the three blue rectangles (the edges of the corresponding peaks in Figure 6) or adopt other less prevalent orientations (e.g., zone B of the C9 map in Figure 6). Nevertheless, as discussed above, it will enable us to be consistent when comparing different systems, as done in section III.2. 


\section{III.1.4 Orientation analysis of the alkyl chains}

We can complete the orientation analysis of the cations by computing probability distributions for angles involving atoms in the alkyl chain. We have computed the distributions for the angle between the surface normal and the vector pointing from the first carbon atom of the butyl chain (C13, cf Figure 2) to the last one (results shown in Figure S1 for the reference system), as well as for the angle between the surface normal and the vector pointing from the nitrogen atom (N1) to the last carbon atom of the butyl chain (results shown in Figure 9). This analysis was performed in density regions that can be characterized mostly by a given ring orientation (Figure 6). To analyze the orientation of the alkyl chains when the rings are in orientations I, II and III we chose the C region of $\mathrm{C} 9$ atoms, the $\mathrm{A}$ region of $\mathrm{C} 2$ atoms and the $\mathrm{C}$ region of $\mathrm{C} 22$ atoms, respectively.

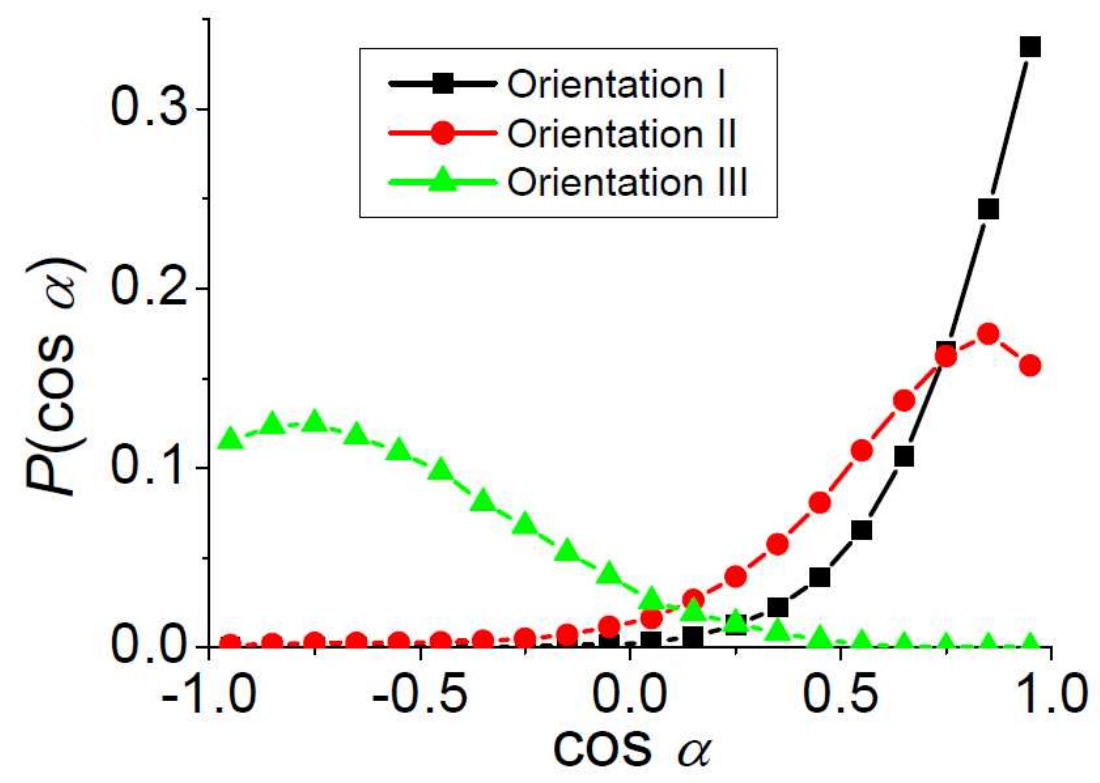

Figure 9 - Probabilities for the angle between the surface normal and the vector pointing from the

$\mathrm{N} 1$ atom to the terminal atom of the butyl chain (C22). Each curve corresponds to a region dominated by one of the preferred ring orientations, as defined by the blue rectangles in Figure 6 .

In orientation I, the butyl chains are mostly pointing directly to the vapor, parallel to the surface normal vector, which translates into the high peak at $\cos \alpha=1\left(\alpha=0^{\circ}\right)$. In zone $\mathrm{A}$ of $\mathrm{C} 2$ atoms, where orientation II is dominant, the chains also tend to stick out to the vapor, but tilted at an angle of about $40^{\circ}$ relative to the surface normal. Because in this orientation the ring is parallel to the surface, it becomes more difficult to rotate the chain such that it points exactly perpendicular to the surface plane. The tilted arrangement provides a compromise between the tendency of the hydrophobic chains to point to the vapor and the energetic penalty for chain rotation. When the butyl chains are on the liquid side, in orientation III, the chains are most likely to form an angle of about $180^{\circ}$ with the surface normal direction (i.e., they point straight into the bulk liquid), but the preference for a given angle is much weaker than for the other two orientations, which indicates a 
relatively large tendency of the chains to coil. This behavior will influence the effect of chain length on the surface structure, which will be analyzed below.

\section{III.1.5 The structure of the interface}

The intrinsic profiles and the orientation analyses presented above provide information of an unprecedented degree of detail about the organization of the IL interface at the molecular level. One question, however, is still unanswered - what is the relative location of each molecular orientation in a global framework. To answer this question, we revisit the global atomic density profiles shown in Figure 3c, but split them into contributions from each orientation. The result of this analysis is shown in Figure 10 for selected representative atoms (the central carbon atom in the cation ring, $\mathrm{C2}$, the terminal carbon in the cation chain, $\mathrm{C} 22$, and the central atom of the anion, P). From the C22 profiles, one can see that the chains in both orientations I and II occupy the ripples of the IL surface (i.e., the regions of the surface that protrude mostly into the vapor phase). As expected, the chains in orientation III are located on the innermost region of the interfacial layer, interacting with the second layer beneath the interface. The $\mathrm{C} 2$ and $\mathrm{P}$ profiles reveal that the ionic moieties are centered practically at the same position regardless of the ring orientation, with, however, a slight tendency for anions and rings that are parallel to the surface (i.e., in orientation II) to be located further on the vapor side. This clearly points to the existence of a tightly packed ionic layer beneath the ripples formed by the non-polar chains, but which also occupies the troughs of the corrugated surface layer.

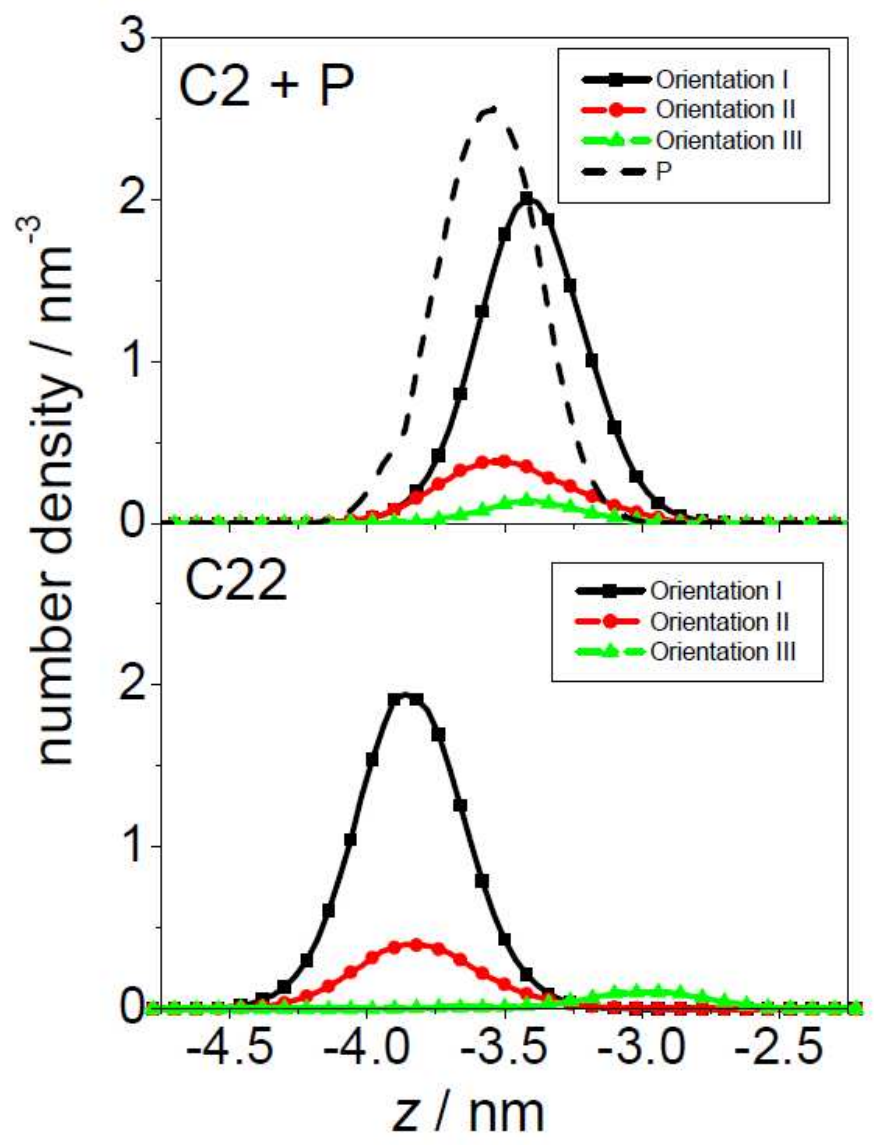


Figure 10 - Global number density profiles for selected atoms belonging to interfacial molecules. The cation profiles are split into contributions arising from each preferential ring orientation, as given by the blue rectangles in Figure 6. The top panel represents profiles belonging to ionic groups (cation ring $\mathrm{C} 2$ atom + anion $\mathrm{P}$ ), while the bottom panel shows profiles describing apolar chains (C22 atom).

A schematic diagram of the proposed structure for the $\mathrm{BMIMPF}_{6}$ interface is shown in Figure 11. In the regions where the surface layer is convex (i.e., the ripples), the alkyl chains are located on the vapor side, followed by the cation rings and the anions on the liquid side (orientation I is dominant). In regions of concave curvature (i.e., the troughs), the ionic groups are mostly facing the vapor phase, with alkyl chains either tilted to the vapor (orientation II) or facing the liquid (orientation III). The result of these molecular arrangements is that the ionic regions of the IL are all concentrated in a tightly packed layer located at almost the same $z$ position on the global frame. This layer is responsible for maintaining the integrity of the liquid phase and causes the density enhancement observed in the global density profiles of Figure 3a. Evidence for the existence of this tightly packed ionic layer was also obtained by Perez-Blanco and Maginn - they studied a different anion ( $\left.\mathrm{NTf}_{2}{ }_{2}{ }^{-}\right)$ and used only a global analysis, but still reported a marked increase in ion pairing with a strong directional character very close to the surface.[41] Our picture of the IL interface, shown in Figure 11 , is conceptually similar to that of other polar liquids, such as water. In fact, it was found that water molecules mostly orient their dipole vectors parallel to the surface, forming a tightly packed and strongly hydrogen-bonded layer, but molecules located in the interface ripples actually point their hydrogen atoms toward the opposite phase.[27;28]

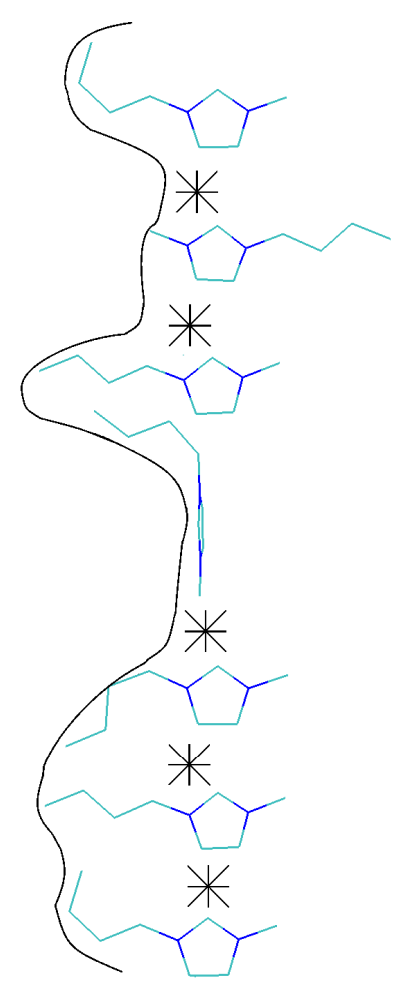


Figure 11 - Diagram depicting the most typical arrangement of IL interfacial molecules, according to our results.

\section{III.2 Comparison of different ILs and conditions}

\section{III.2.1 Effect of the cation alkyl chain length}

Density profiles. In this subsection, results for $\mathrm{EMIMPF}_{6}, \mathrm{BMIMPF}_{6}$ and $\mathrm{HMIMPF}_{6}$ at $360 \mathrm{~K}$ will be compared, in order to assess the effect of the alkyl chain length on the interfacial structure. The obtained global and intrinsic mass density profiles for these three systems are plotted in Figure 12. All density profiles show decreasing bulk density with increasing chains, which is not surprising if one considers these systems as a 1:1 mixture of an organic salt with alkanes of progressively increasing lengths. In other words, all systems contain the same mass of ionic moieties, differing only in the amount of "alkane mass" - increase in the percentage of alkane groups causes decrease in density.

At the interface, each system displays density enhancement (first peak in the global profiles of Figure 12a), which seems to be independent of the alkyl chain length, suggesting that it is mostly caused by the ionic parts of the system (imidazolium ring and anion). What is more, in $\mathrm{HMIMPF}_{6}$ a depleted density region can be observed at about $1-1.5 \mathrm{~nm}$ beyond the first peak of the global profile. A detailed analysis of the number density profiles of individual atoms (Figure S2) reveals that this region is dominated by alkyl chains, while anions and rings are partially squeezed out. On the other hand, this analysis confirms that atoms belonging to cationic rings and anions contribute mostly to regions where a density enhancement is observed. These data confirm the existence of a certain degree of layering in the vicinity of the IL surface (see Figure 11), alternating hydrophobic regions (containing mostly alkyl chains) and polar regions (containing mostly rings and anions), which appears to be more pronounced for longer alkyl chains. Voth and co-workers have also observed an increase in the degree of layering with increasing chain length in their coarse-grained simulations.[19]
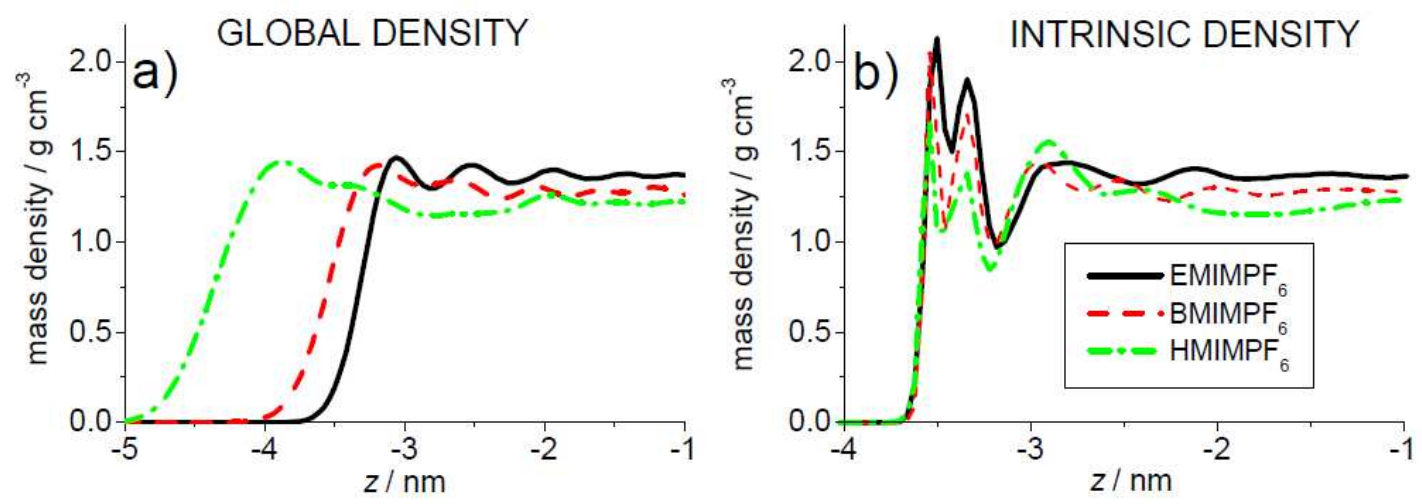

Figure 12 - Global (a) and intrinsic (b) mass density profiles for ILs with different cation alkyl chain lengths at $360 \mathrm{~K}: \mathrm{EMIMPF}_{6}$ - solid black line; $\mathrm{BMIMPF}_{6}$ - dashed red line; $\mathrm{HMIMPF}_{6}$ - dashed-dotted 
green line. The intrinsic profiles are shifted so that the first peak is located at the same position in all the profiles.

In panel b of Figure 12, all intrinsic profiles are shifted along the $z$ axis to the position of the interface of $\mathrm{BMIMPF}_{6}$ at $360 \mathrm{~K}$, so that the sharp peaks of the interfacial sites appear at the same position. An increase in the chain length results in a decrease of the height of the first two interfacial peaks, which are exclusively attributed to interfacial molecules. The position of the second peak compared to the first one, however, is independent of the alkyl chain length. Close inspection of the atomic density profiles revealed that the contribution to the interfacial peaks from both the anions and the rings decreases in absolute terms, compared to an increasing contribution from the alkyl chain atoms. On the other hand, this also means that rings and anions appear less frequently at the interface when the chain length increases, which suggests that the interface becomes more covered by alkyl chains, making it more difficult to "see" rings and anions from the vapor side. This conclusion is in accordance with recent experimental findings. $[9,10]$ Note that the depleted density region on the intrinsic $\mathrm{HMIMPF}_{6}$ density also appears in the intrinsic profile, at $1.5-2.0 \mathrm{~nm}$ beneath the interface. It is interesting to note that the third intrinsic peak of EMIMPF 6 shows already a density very close to the bulk value, whereas for the longer chains this becomes true only at larger depths. This trend suggests that the longer the alkyl chain is, the deeper the effect of the interface propagates into the system.

Ring orientations. The effect of increasing alkyl chain length on the orientation preferences of imidazolium rings was investigated by performing the analysis described in Figure 6 for EMIM, BMIM, and HMIM. As stated above, no qualitative difference was observed on the maps, thus here we turn directly to the quantification of different orientation preferences, using the approach described in section III.1.3.

The obtained percentages for different ring orientations, corresponding to the zones marked by blue rectangles in Figure 6, in $\mathrm{EMIMPF}_{6}, \mathrm{BMIMPF}_{6}$ and $\mathrm{HMIMPF}_{6}$ are listed in Table 2. The increasing alkyl chain length has a clear effect on all orientations - while the percentage of molecules in orientation I increases with increasing chain length, the opposite trend can be observed for orientations II and III. The decreasing occurrence of orientation III can be understood if one considers that the accommodation of chains of increasing size (signifying increasing apolar domains) on the liquid side becomes less favorable. The decreasing amount of orientation II can be understood based on similar principles - a cation in orientation II occupies more surface area than cations in orientation I, thus changing the orientation of a cation from II to I would make it possible to place more alkyl chains on the vapor side. This would also mean that the number of surface cations should increase with increasing chain length, and, indeed, this number increases from $19.6 \%$ to $21.4 \%$ when changing from EMIM to HMIM.

Table 2 - Orientational preferences for ILs with different cation alkyl chain length. Values were obtained by integrating the regions delimited by the blue rectangles in Figure 6 .

System Orientation I Orientation II Orientation III




\begin{tabular}{cccc} 
EMIMPF $_{6}$ & $31.4 \%$ & $13.4 \%$ & $10.0 \%$ \\
\hline BMIMPF $_{6}$ & $45.0 \%$ & $11.2 \%$ & $3.0 \%$ \\
\hline HMIMPF $_{6}$ & $51.9 \%$ & $8.9 \%$ & $1.0 \%$ \\
\hline
\end{tabular}

Chain orientations. Figure 13 shows the chain orientation probabilities for each characteristic ring orientation in systems with different alkyl chain lengths (additional chain orientation plots for all systems are provided in Supporting Information). In orientation I, as described above, most chains are oriented almost precisely along the surface normal, pointing to the vapor. This tendency is independent of the actual length of the chain, and thus the profiles for the three cations in orientation I are identical. In orientation II, the alkyl chains also tend to point mainly to the liquid, but the preferred angle with the interface normal becomes smaller as the chain length increases $\left(\sim 70^{\circ}\right.$ for $\mathrm{EMIM}>\sim 30^{\circ}$ for $\mathrm{BMIM}>0^{\circ}$ for $\left.\mathrm{HMIM}\right)$. Because in this orientation the ring is parallel to the interface, shorter chains are less able to adopt conformations that become perpendicular to the surface, leading to higher average angles. It is noticeable that for EMIM (the most extreme case), the chain can even point to the liquid side in some configurations. In orientation III, the chains are mostly pointing to the liquid side, but they are not always perpendicular to the surface normal instead, the preferred angle is around $130^{\circ}$. This is most likely due to packing constraints on the denser liquid-side of the interface, which disfavor fully extended chain conformations. Longer chains have a higher tendency to coil, giving rise to broader angle distributions. Noticeably, the HMIM distribution even shows a bimodal character, with a significant portion of chains bending back upon themselves and pointing to the vapor. This is yet another manifestation of the polar/apolar layering of the IL, which drives the hydrophobic moieties to the vapor phase. 


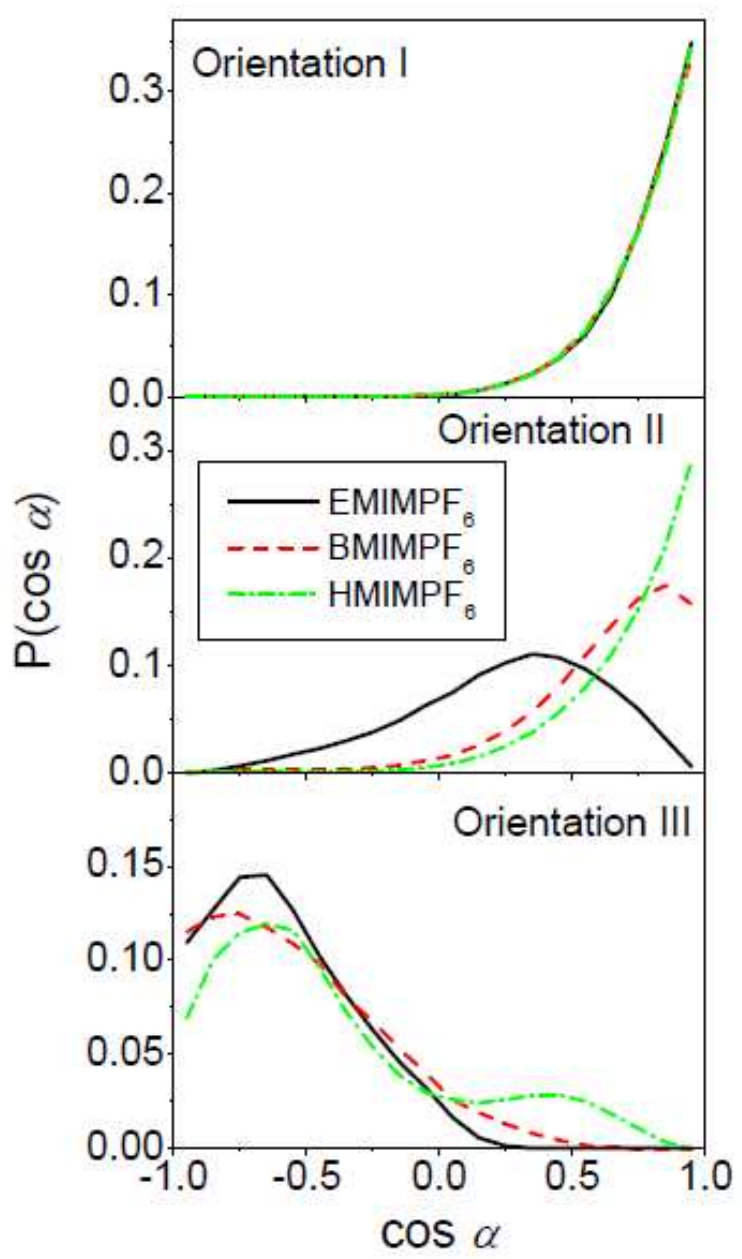

Figure 13 - Probabilities for the angle between the surface normal and the vector pointing from the $\mathrm{N} 1$ atom to the terminal atom of the alkyl chain (C16, C22 and C28 for EMIM, BMIM and HMIM, respectively) in ILs with different cation alkyl chain length. The top panel is for rings in orientation I, the middle panel for orientation II and the lower panel for orientation III, as defined by the blue rectangles in Figure 6.

In summary, as the cation alkyl chain length increases, the surface layer of the IL becomes more populated by non-polar alkyl groups, which have increasing tendency to point straight into the vapor phase. These alkyl moieties increasingly "cover" the polar parts of the IL (the rings and the anions), making the surface progressively more similar to that of an alkane, and thus lowering the surface tension. Recent simulations by Pensado et al. also show a decrease in surface tension with increase in chain length.[23] Moreover, based on the trend observed in Table 2, one may speculate that as the chain length increases beyond a critical size, orientations II and III will become residual and the surface will be completely covered by alkyl groups. This is precisely the trend that is observed experimentally, where the surface tension is seen to decrease with chain length, but eventually becomes constant for sufficiently long chains.[42] At this point, only a qualitative comparison can be 
carried out, but further studies are underway to enable a more quantitative comparison between simulations and experiments.

\section{III.2.2 Effect of changing anion}

Density profiles. When discussing the effect of changing the anion, one has to bear in mind that it implies two major effects: a geometric (steric) effect caused by changes in molecular size and/or shape, and an energetic effect, caused by changes in the local charge density. For this comparison we have chosen only highly symmetric monovalent anions to eliminate the effects of the shape and of the amount of charge. We are thus practically changing only the size and the charge density of the anion. Figure 14 shows global (panel a) and intrinsic (panel b) density profiles obtained in $\mathrm{BMIMCl}$, $\mathrm{BMIMBF}_{4}$ and $\mathrm{BMIMPF}_{6}$ at $360 \mathrm{~K}$.
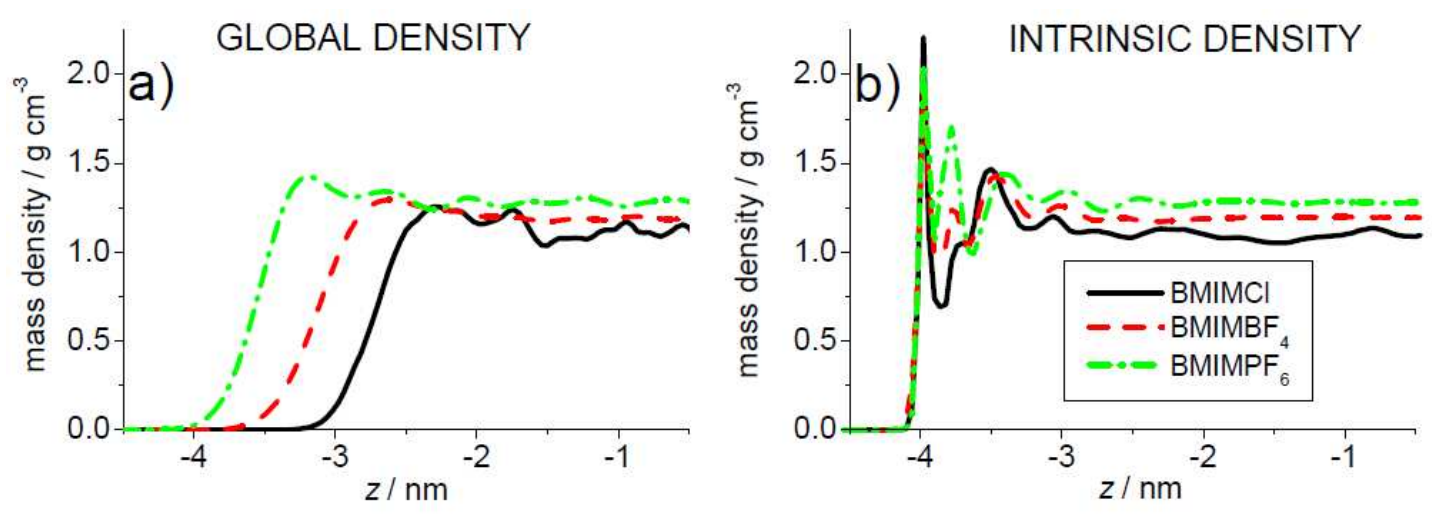

Figure 14 - Global (a) and intrinsic (b) mass density profiles for ILs with different anions at $360 \mathrm{~K}$ : $\mathrm{BMIMCl}$ - solid black line; $\mathrm{BMIMBF}_{4}$ - dashed red line; $\mathrm{BMIMPF}_{6}$ - dashed-dotted green line. The intrinsic profiles are shifted so that the first peak is located at the same position in all the profiles.

Panel a of Figure 14 shows a monotonic global mass density increase, both at the interface and in the bulk, with increasing anion size. In line with the reasoning presented in the comparison of different alkyl chain lengths, one can reasonably suppose that this density change is due to the fact that increasing anion mass will decrease the relative weight of alkyl chains in the system, thus it will decrease the "dilution" of the system. The comparison of intrinsic profiles shows several interesting aspects. The first peak (due to surface atoms) is more or less of the same height in each profile, but the second peak undergoes a significant change - it is quite pronounced in $\mathrm{BMIMPF}_{6}$, becomes much smaller in $\mathrm{BMIMBF}_{4}$, until it appears only as a shoulder of the third peak in the BMIMCl profile. The examination of the number density profiles of individual atoms in $\mathrm{BMIMPF}_{6}$ shows that atoms that are separated by one or two (or sometimes three) bonds from surface atoms give the most important contribution to the second peak, thus typically $P$, non-interfacial $F, C 19$ and C16 atoms. In $\mathrm{BMIMBF}_{4}$ the peak of $\mathrm{B}$ atoms appears closer to the interface whereas the contribution of carbon atoms moves to larger distance from the interface. In $\mathrm{BMIMCl}$, as the anion has only one site, the contribution from the interfacial anions falls entirely on the interface. Thus the largest contribution 
to the second peak disappears, which makes the peak become just a shoulder. Interestingly, though, the third peak has roughly the same height in each system regardless of the anion.

It should also be noted that density oscillations in the global profiles were much more prominent in the $\mathrm{BMIMCl}$ system than in the other two. The intrinsic density profiles calculated on the two sides of the liquid slab were not precisely symmetric (the orientation distributions, discussed below, also showed some degree of asymmetry). These signs suggest that the BMIMCl system may not have reached full equilibrium, as already mentioned in section II.1. Nevertheless, the results we obtained can still be used for discerning meaningful qualitative trends as the anions size is changed.

Ring orientations. A small but noticeable effect arises in the orientation maps when decreasing the anion size - rings in orientation II have less freedom to tilt around the NN axis. The semi-quantitative analysis of the orientation preferences (Table 3) reveals that changes in the occurrence of orientation I are barely noticeable, while orientation III decreases in importance with decreasing anion size and orientation II increases in importance. Together with these orientation changes, the percentage of cations located at the surface increases from $20.3 \%$ to $27.8 \%$ when the anion changes from $\mathrm{PF}_{6}{ }^{-}$to $\mathrm{Cl}^{-}$, while the percentage of surface anions decreases. This can be partially explained based on geometric arguments - smaller anions leave more room at the surface for additional cations and ring orientations that occupy more surface area (i.e., orientation II). Moreover, anions appear at the interface mostly to counterbalance the repulsion between interfacial cations. Decreasing anion size with the same charge results in higher charge density, which implies that fewer small anions are needed to stabilize the interface than larger ones. This is corroborated by the fact that the intrinsic charge density distribution shows noticeably larger negative charge at the interface in $\mathrm{BMIMPF}_{6}$ than in $\mathrm{BMIMCl}$ (see Figure S4).

Table 3 - Orientational preferences for ILs with different anions. Values were obtained by integrating the regions delimited by the blue rectangles in Figure 6.

\begin{tabular}{cccc} 
System & Orientation I & Orientation II & Orientation III \\
\hline $\mathrm{BMIM} \mathrm{Cl}$ & $43.3 \%$ & $20.5 \%$ & $0.6 \%$ \\
\hline $\mathrm{BMIM} \mathrm{BF}_{4}$ & $41.8 \%$ & $16.5 \%$ & $1.4 \%$ \\
\hline $\mathrm{BMIM} \mathrm{PF}_{6}$ & $45.0 \%$ & $11.2 \%$ & $3.0 \%$ \\
\hline
\end{tabular}

Chain orientations. The chain orientation distributions for orientations I and II (Figure 15) do not show much sensitivity to the anion size. Nevertheless, a slight tendency for increased order with decreasing anion size can be discerned in both plots. In orientation III, a pronounced change can be observed in $\mathrm{BMIMCl}$, with the appearance of a second peak corresponding to chains bending back and pointing into the vapor (analogous to what was seen above for $\mathrm{HMIMPF}_{6}$ ). One must not over interpret this result, on the one hand because the $\mathrm{BMIMCl}$ system experienced equilibration difficulties (see section II.1), and on the other hand because only a small fraction of $\mathrm{BMIMCl}$ chains actually adopt orientation III (see Table 3), leading to poor statistical sampling. Nevertheless, this effect may be due to a more closely packed ionic layer beneath the interface, brought about by the 
higher charge density (and consequently stronger electrostatic anion-ring interactions) in ILs with smaller anions.

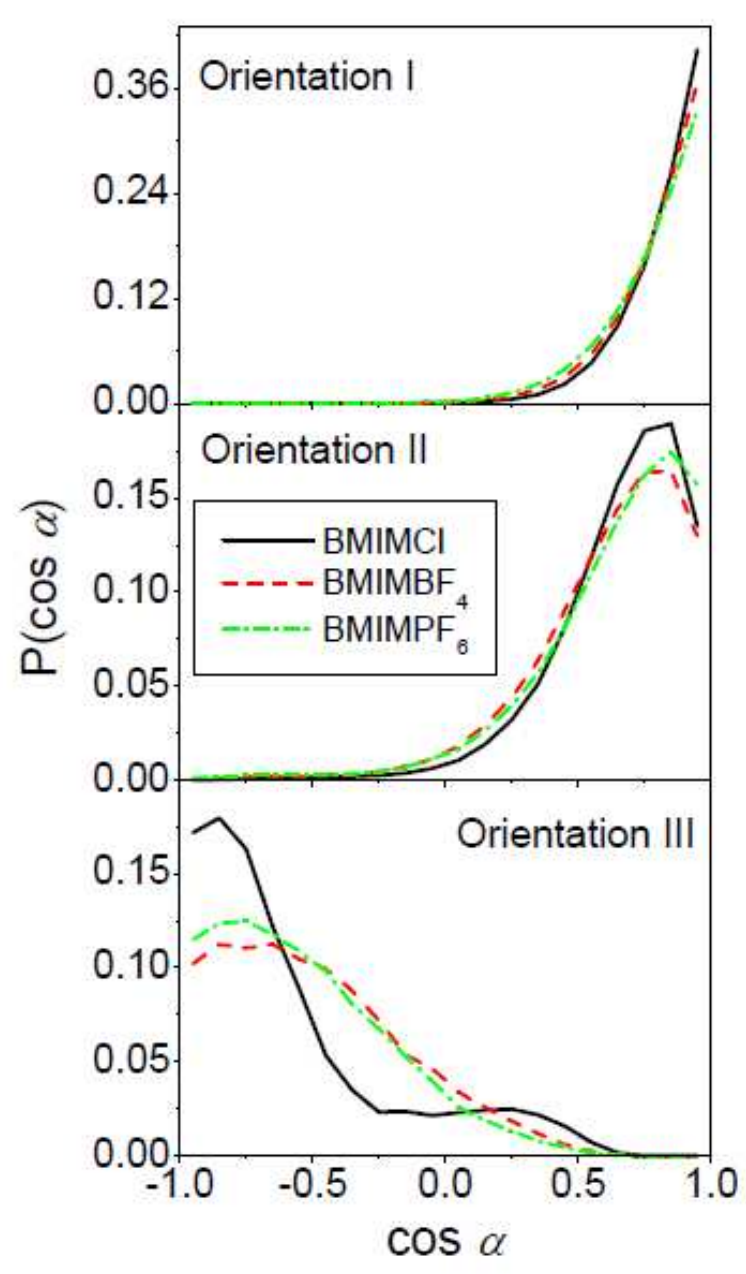

Figure 15 - Probabilities for the angle between the surface normal and the vector pointing from the $\mathrm{N} 1$ atom to the terminal atom of the butyl chain (C22) in ILs with different anions. The top panel is for rings in orientation I, the middle panel for orientation II and the lower panel for orientation III, as defined by the blue rectangles in Figure 6 .

To summarize, our results suggest that as the anion size decreases (everything else remaining constant), the ionic layer at the surface becomes more tightly packed and somewhat more rigid. This promotes an increase in the degree of ordering at the surface and thus reduces the orientational freedom of the cations, which adopt orientations that deviate less from the preferred arrangements. Conversely, larger anions increase the amount of free space in the vicinity of the interface, and thus enable some of the alkyl chains of surface cations to be located on the liquid side of the interface. The observation that larger anions increase the amount of voids in the bulk IL structure is in agreement with our interpretation.[16] 


\section{III.2.3 Effect of temperature}

Density profiles. The global (panel a) and intrinsic (panel b) density profiles for BMIMPF 6 obtained at $298 \mathrm{~K}, 330 \mathrm{~K}$ and $360 \mathrm{~K}$ are plotted in Figure 16. Based on the density profiles, we can establish that temperature has only a small effect on the system at least in the range investigated. Global profiles show slightly decreasing density at the interface and in the bulk, due to thermal expansion, as well as somewhat smoother profiles when the temperature rises. The intrinsic density profiles in panel $b$ suggest very similar behavior and structure; however, the first two intrinsic density peaks (exclusively attributed to surface molecules) are slightly sharper at room temperature than at higher temperatures. The fact that the first two intrinsic peaks are practically indistinguishable at the two highest temperatures studied here suggests that the interfacial structure is very similar at these two temperatures, while one should expect a higher degree of order at room temperature.
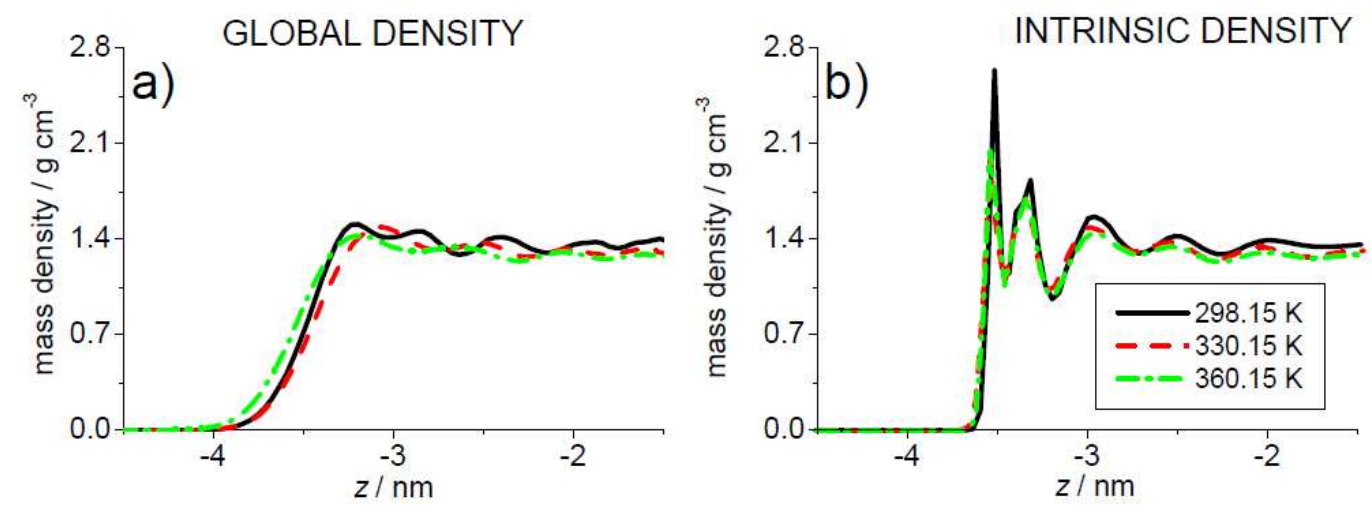

Figure 16 - Global (a) and intrinsic (b) mass density profiles for BMIMPF $_{6}$ at different temperatures: $298 \mathrm{~K}$ - solid black line; $330 \mathrm{~K}$ - dashed red line; $360 \mathrm{~K}$ - dashed-dotted green line. The intrinsic profiles are shifted so that the first peak is located at the same position in all the profiles.

Ring orientations. When looking at the change in frequencies of the ring orientations, one can notice small but clear differences as is seen in Table 4. As hinted by the density profiles, prevalences for the different orientations are very similar at the two highest temperatures, while they show more pronounced differences at room temperature. In general, it can be said that increasing temperature decreases the percentage of orientations II and III, while the dominance of orientation I increases. Thus, it seems that increasing temperature disfavors both rings lying on the surface plane and alkyl chains pointing to the liquid phase. Nevertheless, one should interpret these results with caution, since the trends observed in Table 4 are not completely clear.

Table 4 - Orientational preferences for $\mathrm{BMIMPF}_{6}$ at different temperatures. Values were obtained by integrating the regions delimited by the blue rectangles in Figure 6.

\begin{tabular}{cccc} 
System & Orientation I & Orientation II & Orientation III \\
\hline $298.15 \mathrm{~K}$ & $35.5 \%$ & $16.9 \%$ & $5.3 \%$ \\
\hline $330.15 \mathrm{~K}$ & $51.3 \%$ & $9.1 \%$ & $2.0 \%$ \\
\hline
\end{tabular}


Chain orientations. In the studied range, the temperature has no noticeable effect on the orientation preference of chains pointing to the vapor (Figure 17). However, a trend can be observed for the chains pointing to the bulk liquid - the peak for the most frequent orientation shifts closer to the direction of the surface normal with increasing temperature. At lower temperatures, chains in the most probable orientation form an angle of around $125^{\circ}$ with the surface normal, while at $360 \mathrm{~K}$, the most probable angle is about $145^{\circ}$. This can be interpreted by considering the enhanced degree of structure at low temperature observed in the density profiles of Figure 16. Thus, at low temperature the interfacial layer becomes more tightly packed, which forces the chains that are pointing into the liquid to show a higher probability of coiling. In other words, steric effects caused by liquid packing are overcoming the energetic penalty that the chains must pay to adopt less favorable coiled configurations.

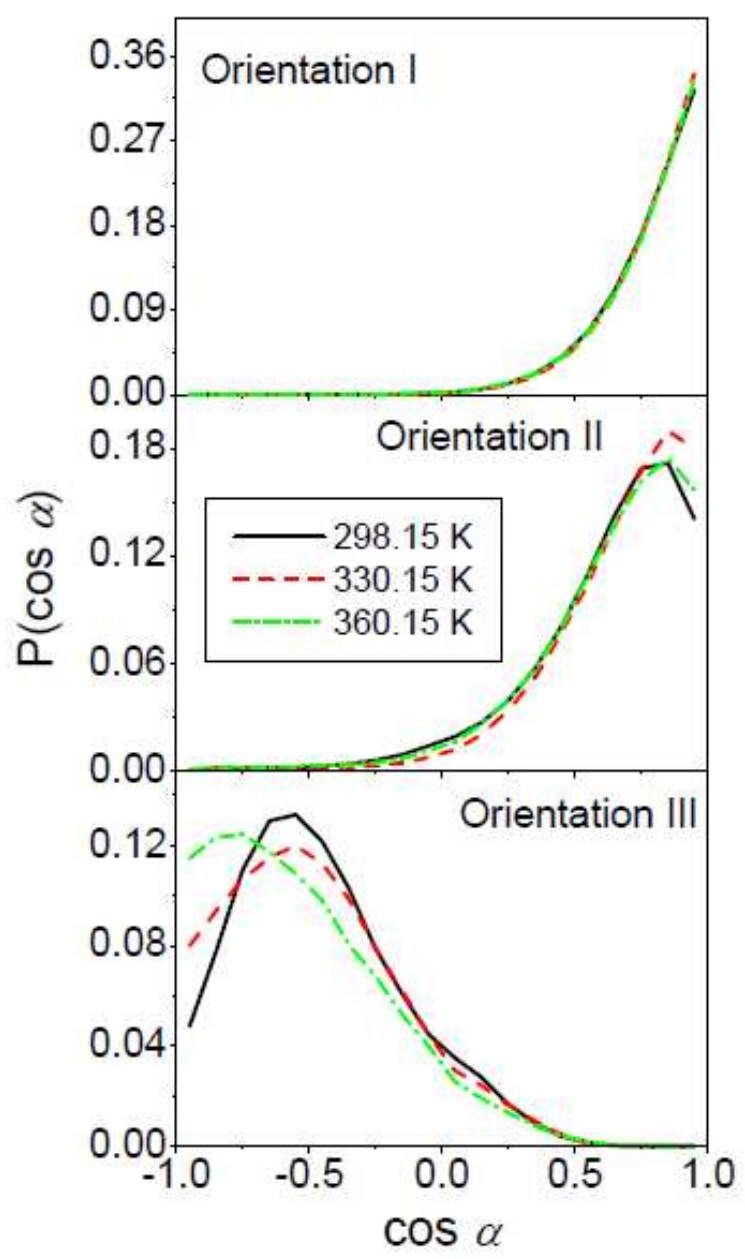

Figure 17 - Probabilities for the angle between the surface normal and the vector pointing from the $\mathrm{N} 1$ atom to the terminal atom of the butyl chain (C22) in $\mathrm{BMIMPF}_{6}$ at different temperatures. The 
top panel is for rings in orientation I, the middle panel for orientation II and the lower panel for orientation III, as defined by the blue rectangles in Figure 6.

\section{III.2.4 Effect of different molecular models}

Density profiles. Global and intrinsic density profiles obtained in $\mathrm{BMIMPF}_{6}$ at $298 \mathrm{~K}$ with the CLaP, BB, and Liu 2011 models are plotted in Figure 18. The comparisons of the densities, in general, do not suggest large qualitative changes in the structure; however, some features can be unequivocally identified. The global profiles show that the two reduced charge models (BB and Liu 2011) display smoother density profiles, in accordance with their lower viscosity compared to the CLaP model. It can also be seen that the density enhancement is somewhat larger with the Liu 2011 model than with the other two. These differences are even more pronounced in the intrinsic density profiles, where the profile determined with the Liu 2011 model shows two interfacial peaks that are much sharper than with the other two models. One can also note that the position of the third peak obtained with this model is somewhat shifted toward the interface. This indicates a stronger degree of packing in the interfacial region in intrinsic terms, and can probably also explain the higher global mass density enhancement observed with the Liu 2011 model at the interface. Because there are several different parameters between the Liu 2011 model and the other two (see Section II.1), it is difficult to clearly establish the reason for these differences. However, they are likely to be related to the united-atom representation of the alkyl chains - chains of simpler shape and smaller size are easier to pack. The very sharp profiles of individual atoms obtained with the Liu 2011 model also corroborate this interpretation. Finally, it is also important to notice that the intrinsic profiles for CLaP and BB are almost alike. The most important difference between these models is the total charge on each ion (lower in the case of $\mathrm{BB}$ ), which suggests that this parameter does not have a significant influence on the density profiles.
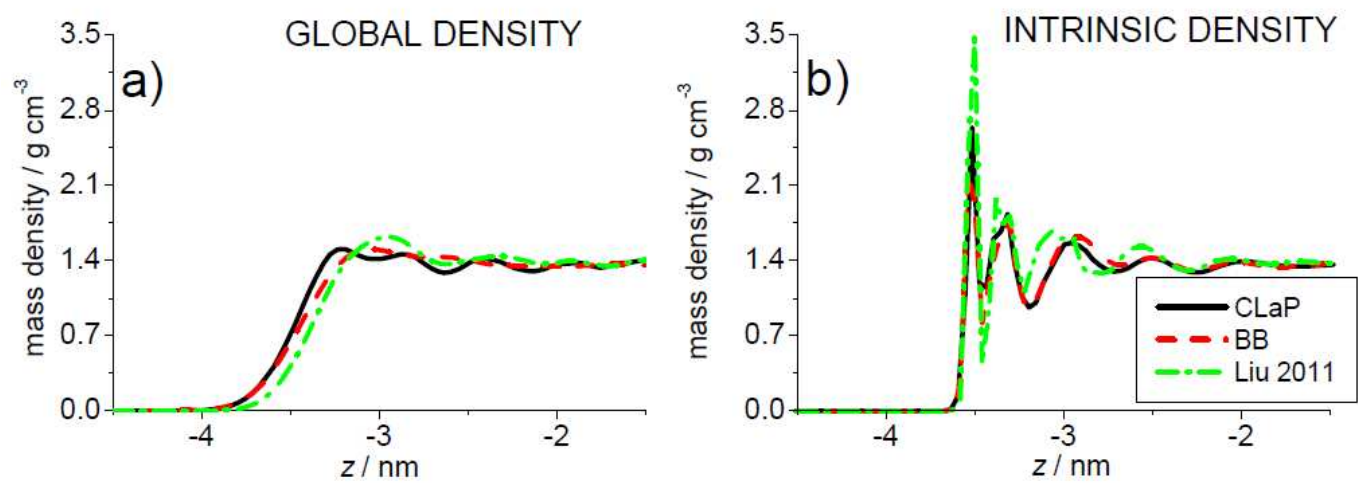

Figure 18 - Global (a) and intrinsic (b) mass density profiles for $\mathrm{BMIMPF}_{6}$ at $298 \mathrm{~K}$ using different molecular models: CLaP - solid black line; BB - dashed red line; Liu 2011 - dashed-dotted green line.

The intrinsic profiles are shifted so that the first peak is located at the same position in all the profiles. 
Ring orientations. The differences between the models are more significant in the molecular orientations at the interface (see Table 5 for the ring orientation prevalences). Switching from the CLaP model to the BB model, which has basically the same geometrical properties but reduced charges, a strong increase in orientation I can be observed besides a decrease in orientations II and III. This suggests that the arrangement of the cation rings on the surface is strongly sensitive to electrostatic effects. Moving to the Liu 2011 model, where both the charge distribution and the geometry of the cations are different, the fraction of orientation I increases significantly, while the occurrence of orientation II decreases and orientation III increases compared to the BB model. This can be the result of an interplay between several effects that cannot be fully elucidated based on the present study. However, these results clearly illustrate that the choice of molecular model can have an important effect when describing interfacial properties of ionic liquids.

In this context, it is useful to compare the surface tensions obtained by each model with the experimental value of $44 \mathrm{mN} / \mathrm{m}$ [43]. Using the pressure tensor method [44], we obtained $67 \pm 5$ $\mathrm{mN} / \mathrm{m}$ for $\mathrm{CL}, 35 \pm 3$ for $\mathrm{BB}$ and $38 \pm 4$ for Liu 2011. Although the data suffers from large statistical errors, it is clear that the $\mathrm{CL}$ model significantly overestimates the surface tension, while the other two models do a fairly good job. This may be due to the fact that both BB and Liu 2011 use rescaled charges on each ion, which are known to improve the accuracy of ionic liquid models [34,45]. A systematic comparison between experimental and simulated interfacial tensions for a range of different ionic liquids modeled using different approaches would be necessary to further clarify this issue.

Table 5 - Orientational preferences for $\mathrm{BMIMPF}_{6}$ at $298 \mathrm{~K}$ using different molecular models. Values were obtained by integrating the regions delimited by the blue rectangles in Figure 6.

\begin{tabular}{cccc} 
System & Orientation I & Orientation II & Orientation III \\
\hline CLaP & $35.5 \%$ & $16.9 \%$ & $5.3 \%$ \\
\hline BB & $44.0 \%$ & $8.9 \%$ & $1.6 \%$ \\
\hline Liu 2011 & $63.2 \%$ & $4.7 \%$ & $3.6 \%$ \\
\hline
\end{tabular}

Chain orientations. The comparison of the chain orientation preferences obtained with different models shows (Figure 19) that in orientations I and II the CLaP and BB models give practically the same distributions of chain orientations. This is somewhat expected, since these models practically only vary in the total ionic charge, which is unlikely to affect the arrangement of non-polar chains on the vapor side. However, when the chains are on the liquid side (i.e., in orientation III), differences between these two models appear. Again, this is likely due to a change in the degree of packing of the ionic moieties of the IL - the CLaP model, with higher charge, induces more pronounced ionic packing, leading to higher tendency of the chains to coil. As for the Liu 2011 model, the steeper distributions for orientations I and III are probably a consequence of an increased stiffness of the united atom alkyl chains in this model. 


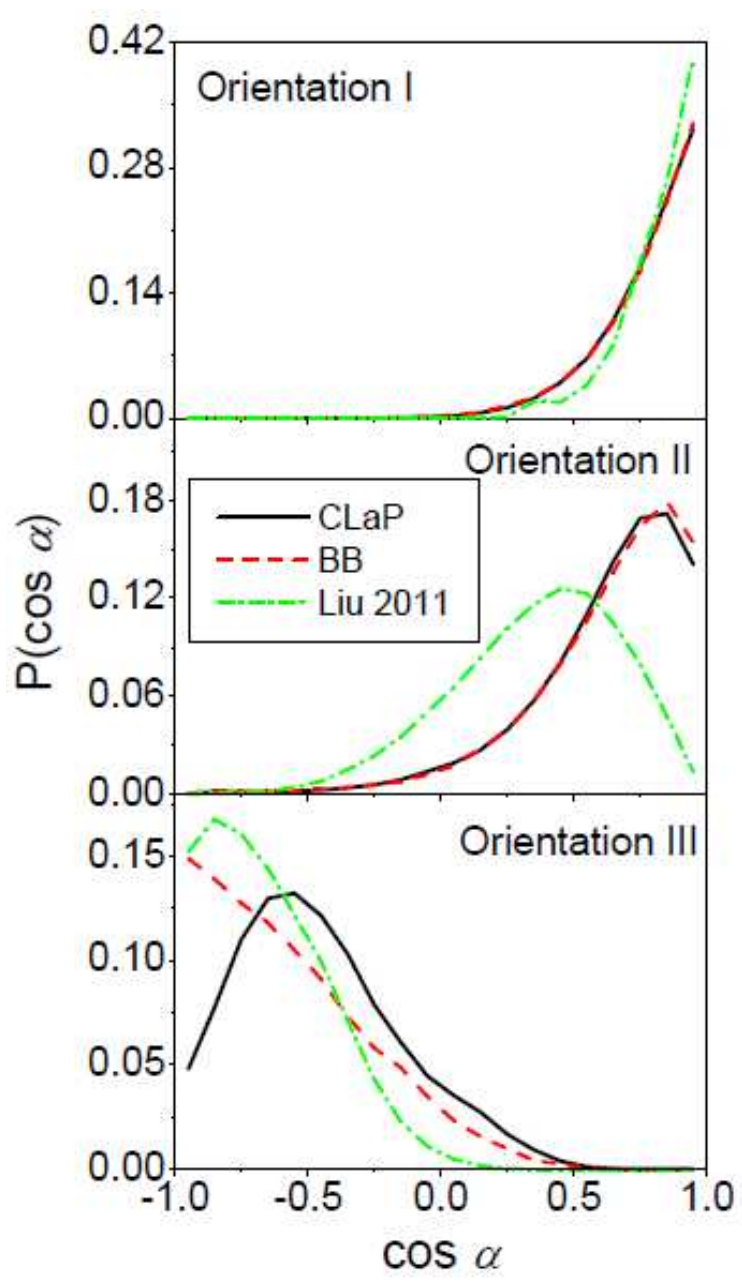

Figure 19 - Probabilities for the angle between the surface normal and the vector pointing from the $\mathrm{N} 1$ atom to the terminal atom of the butyl chain (C22) in $\mathrm{BMIMPF}_{6}$ at $298 \mathrm{~K}$ using different molecular models. The top panel is for rings in orientation I, the middle panel for orientation II and the lower panel for orientation III, as defined by the blue rectangles in Figure 6.

\section{Conclusions}

We have presented a systematic molecular simulation study of different vapor/liquid interfaces of pure ionic liquids of the imidazolium family. By combining intrinsic analysis methods with bivariate orientation analysis, we were able to obtain a detailed picture of the molecular-level structure of these interfaces, and identify the most predominant molecular arrangements. The anisotropy caused by phase separation induces a strong degree of molecular ordering at the surface of the liquid, whereby alternating layers of non-polar (alkyl chains) and polar (imidazolium rings and anions) regions are formed. Specifically, we observed that the ripples of the corrugated surface layer are mostly populated by alkyl chains that extend directly into the vapor phase. This is followed by a dense and tightly packed ionic layer, responsible for the density enhancement observed in a global 
analysis framework. Parts of this ionic layer occupy the troughs of the corrugated surface, and are thus "visible" from the vapor phase. An additional layer dominated by alkyl chains exists on the liquid side, until eventually this layering dies out as we approach the bulk liquid phase.

We have systematically assessed the effect of cation chain length, anion size, temperature and choice of molecular model on the structure of the surface. In general, the response of the interfacial structure results from a balance between geometric and energetic effects. Increasing alkyl chain lengths generally increase the tendency of chains to point to the vapor, which consequently increases the coverage of the surface by alkyl groups. The degree of layering also extends further into the liquid as the chains become longer. Increasing anion size tends to promote a disruption of the tightly packed ionic layer. As a consequence, cations have increased orientational freedom, and more chains are able to point to the liquid side. Similarly, cation rings occupying more surface area are favored by increasing anion size. Temperature seems to have a negligible influence on the overall density profiles and a relatively small effect on molecular orientations.

Our results also show that the choice of molecular model may have a strong effect on the molecular arrangements at the surface, even if the effect on the overall density is small. Further studies are needed to decide which characteristics of the model have the most important impact in these properties, and to clearly establish which models are better at quantitatively describing interfacial properties.

Finally, our study demonstrates that interfacial properties may be tuned to meet a desired goal by judiciously choosing the appropriate anion and cation of the ionic liquid. For example, by increasing the cation chain length one may increase the affinity of the surface for non-polar solutes, enhancing their adsorption at the interface. Also, choosing a larger anion will "loosen" the surface layer, thereby likely reducing the resistance to solute absorption by the ionic liquid. In this sense, molecular simulation studies of solute transfer across IL interfaces may be an interesting avenue to explore in the near future.

\section{Supporting Information}

Additional figures that complement the discussion in the main paper: chain orientation probabilities, charge density profiles, atomic density profiles.

\section{Acknowledgements}

This work is supported by projects PTDC/EQU-FTT/104195/2008, PEst-C/EQB/LA0020/2011 and Pest-C/EQB/LA0006/2011, financed by FEDER through COMPETE - Programa Operacional Factores de Competitividade and by FCT - Fundação para a Ciência e a Tecnologia. G. H. acknowledges a research grant from FCT with the reference SFRH/BPD/73891/2010. The authors gratefully acknowledge helpful discussions with Vitaly V. Chaban during the preparation of simulations. 


\section{References}

[1] Ionic liquids as Green Solvents. Progress and Prospects, ed. R. D. Rogers and K. R. Seddon, Americal Chemical Society, Washington DC, 2003.

[2] Ionic Liquids in Synthesis, ed. T. Welton and P. Wasserscheid, VCH-Wiley Weinheim, Germany, $2^{\text {nd }}$ edition, 2007.

[3] H. Watarai, Trends. Anal. Chem., 1993, 12, 313.

[4] L. A. Blanchard, D. Hancu, E. J. Beckman, J. F. Brennecke, Nature, 1999, 28, 399.

[5] M Gratzel, J. Photochem. Photobiol. A, 2004, 164, 3.

[6] T. Welton, Chem. Rev., 1999, 99, 2071.

[7] P. Hapiot, C. Lagrost, Chem. Rev., 2008, 108, 2238.

[8] K. R. Seddon, J. Chem. Technol. Biotechnol., 1997, 68, 351.

[9] C. Ridings, V. Lockett and G. Andersson, Phys. Chem. Chem. Phys., 2011, 13, 17177.

[10] J. R. Roscioli and D. J. Nesbitt, J. Phys. Chem. A, 2011, 115, 9764.

[11] T. Iwahashi, T. Nishi, H. Yamane, T. Miyamae, K. Kanai, K. Seki, D. Kim and Y. Ouchi, J. Phys. Chem. C, 2009, 113, 19237.

[12] C. Waring, P. A. J. Bagot, J. M. Slattery, M. L. Costen and K. G. McKendrick, J. Phys. Chem. A, 2010, 114 (14), 4896.

[13] C. S. Santos and S. Baldelli, Chem. Soc. Rev., 2010, 39, 2136.

[14] Computer Simulation of Liquids, M. P. Allen and D. J. Tildesley, Oxford University Press, 1989.

[15] C. Cadena, J. L. Anthony, J. K. Shah, T. I. Morrow, J. F. Brennecke, and E. J. Maginn, J. Am. Chem. Soc., 2004, 126, 5300.

[16] B. Qiao, C. Krekeler, R. Berger, L. Delle Site, and C. Holm, J. Phys. Chem. B, 2008, 112, 1743.

[17] L. X. Dang and C. D. Wick, J. Phys. Chem. B, 2011, 115, 6964.

[18] S. S. Sarangi, S. G. Raju and S. Balasubramanian, Phys. Chem. Chem. Phys., 2011, 13, 2714.

[19] W. Jiang, Y. Wang, T. Yan and G. A. Voth, J. Phys. Chem. C, 2008, 112, 1132.

[20] R. M. Lynden-Bell, J. Kohanoff and M. G. del Pópolo, Faraday Discuss., 2005, 129, 57.

[21] R. M. Lynden-Bell, Mol. Phys., 2003, 101, 2625.

[22] G. Hantal, M. N. D. S. Cordeiro and M. Jorge, Phys. Chem. Chem. Phys., 2011, 13, 21230. 
[23] A. S. Pensado, M. F. Costa Gomes, J. N. Canongia Lopes, P. Malfreyt, A. A. H. Pádua, Phys. Chem. Chem. Phys., 2011, 13, 13518.

[24] F. P. Buff, R. A. Lovett and F. H. Stillinger Jr, Phys. Rev. Lett., 1965, 15, 621.

[25] E. Chacón and P. Tarazona, Phys. Rev. Lett., 2003, 91, 166103.

[26] J. Chowdhary and B. M. Ladanyi, J. Phys. Chem. B, 2006, 110, 15442.

[27] M. Jorge and M. N. D. S. Cordeiro, J. Phys. Chem. C, 2007, 111, 17612.

[28] L. B. Pártay, G. Hantal, P. Jedlovszky, A. Vincze and G. Horvai, J. Comput. Chem., 2008, 29, 945.

[29] E. Lindhal, B. Hess and D. van der Spoel, J. Mol. Mod., 2001, 7, 306.

[30] G. Bussi, D. Donadio and M Parrinello, J. Chem. Phys., 2007, 126, 014101.

[31] U. Essman, L. Perera, M. L. Berkowitz, T. Darden, H. Lee and L. G. Pedersen, J. Chem. Phys, $1995,103,8577$.

[32] J. P. Ryckaert, G. Ciccotti and H. J. C. Berendsen, J. Comput. Phys., 1977, 23, 327.

[33] J. N. Canongia Lopes, J. Deschamps and A. A. H. Pádua, J. Phys. Chem. B, 2004, 108, 2038 ; J. N. Canongia Lopes, J. Deschamps and A. A. H. Pádua, J. Phys. Chem. B, 2004, 108, 11250.

[34] B. L. Bhargava and S. Balasubramanian, J. Chem. Phys., 2007, 127, 114510.

[35] X. Zhong, Z. Liu and D. Cao, J. Phys. Chem. B, 2011, 115, 10027.

[36] Z. Liu, S. Huang and W. Wang, J. Phys. Chem. B, 2004, 108, 12978.

[37] M. Jorge, P. Jedlovszky and M. N. D. S. Cordeiro, J. Phys. Chem. C, 2010, 114, 11169.

[38] M. Jorge, G. Hantal, P. Jedlovszky and M. N. D. S. Cordeiro, J. Phys. Chem. C, 2010, 114, 18656.

[39] G. Hantal, M. Darvas, L. B. Pártay, G. Horvai and P. Jedlovszky, J. Phys.: Condens. Matter, 2010, 22, 284112.

[40] P. Jedlovszky, A. Vincze, G. Horvai, J. Chem. Phys., 2002, 117, 2271.

[41] M. E. Perez-Blanco, E. J. Maginn, J. Phys. Chem. B, 2011, 115, 10488.

[42] P. J. Carvalho, M. G. Freire, I. M. Marrucho, A. J. Queimada, J. A. P. Coutinho, J. Chem. Eng. Data 2008, 53, 1346.

[43] J. Klomfar, M. Souckova, J. Patek, J. Chem. Eng. Data, 2009, 54, 1389. 
[44] J. Rowlinson, B. Widom, Molecular Theory of Capillarity; Clarendon Press: Oxford, U. K., 1982.

[45] T. I. Morrow, E. J. Maginn, J. Phys. Chem. B, 2002, 106, 12807. 


\section{TABLE OF CONTENTS ENTRY}

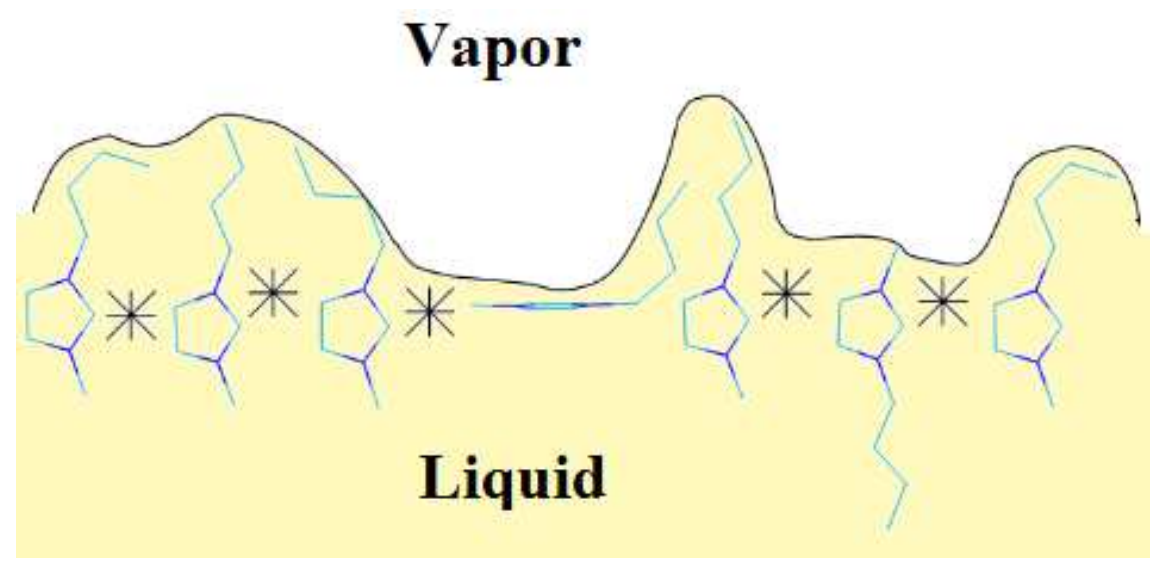

The vapour-liquid interfaces of several ionic liquids were studied in detail using molecular simulation and novel intrinsic analysis methods. 


\section{Supporting information}

A systematic molecular simulation study of ionic liquid surfaces using intrinsic analysis methods

\section{György Hantal ${ }^{1,2}$, luliia Voroshylova², M. Natália D S Cordeiro ${ }^{2}$ and Miguel Jorge $^{1, *}$}

${ }^{1}$ LSRE - Laboratory of Separation and Reaction Engineering - Associate Laboratory LSRE/LCM, Faculdade de Engenharia, Universidade do Porto, Rua Dr. Roberto Frias, 4200-465 Porto, Portugal

${ }^{2}$ REQUIMTE - Associate Laboratory, Faculdade de Ciências, Universidade do Porto, Rua do Campo Alegre, 687, 4169-007 Porto, Portugal

Additional Figures obtained from the intrinsic analysis and orientation analysis of the ionic liquid interfaces.

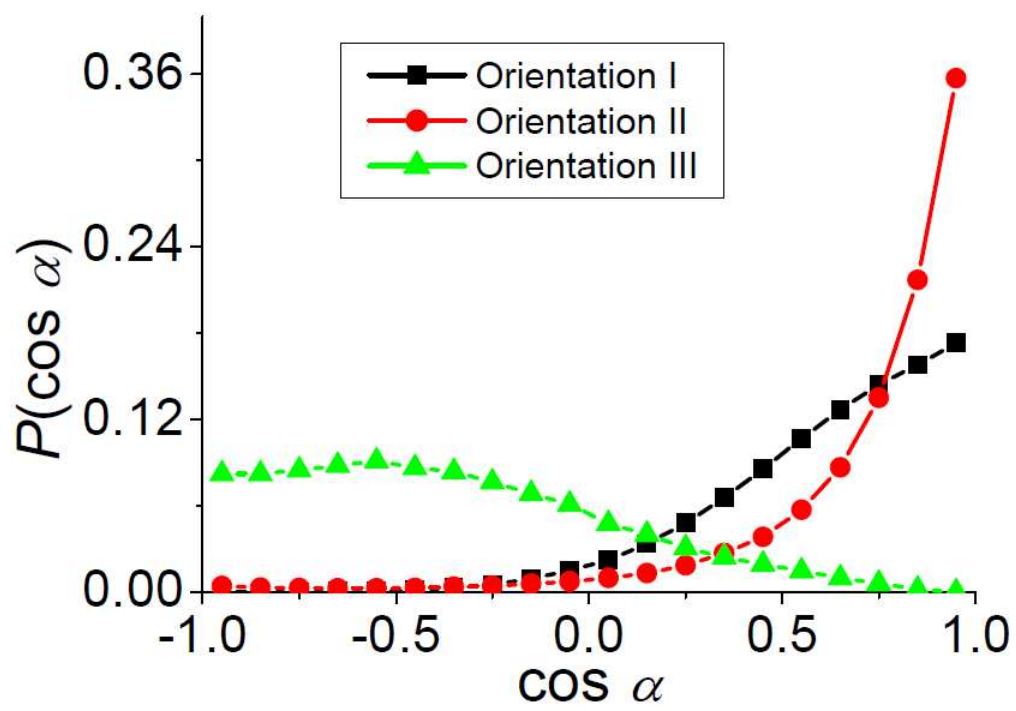

Figure S1 - Probabilities for the angle between the surface normal and the vector pointing from the first carbon atom of the butyl chain (C13) to the terminal one (C22). Each curve corresponds to a region dominated by one of the preferred ring orientations, as defined by the blue rectangles in

Figure 6 of the paper. 


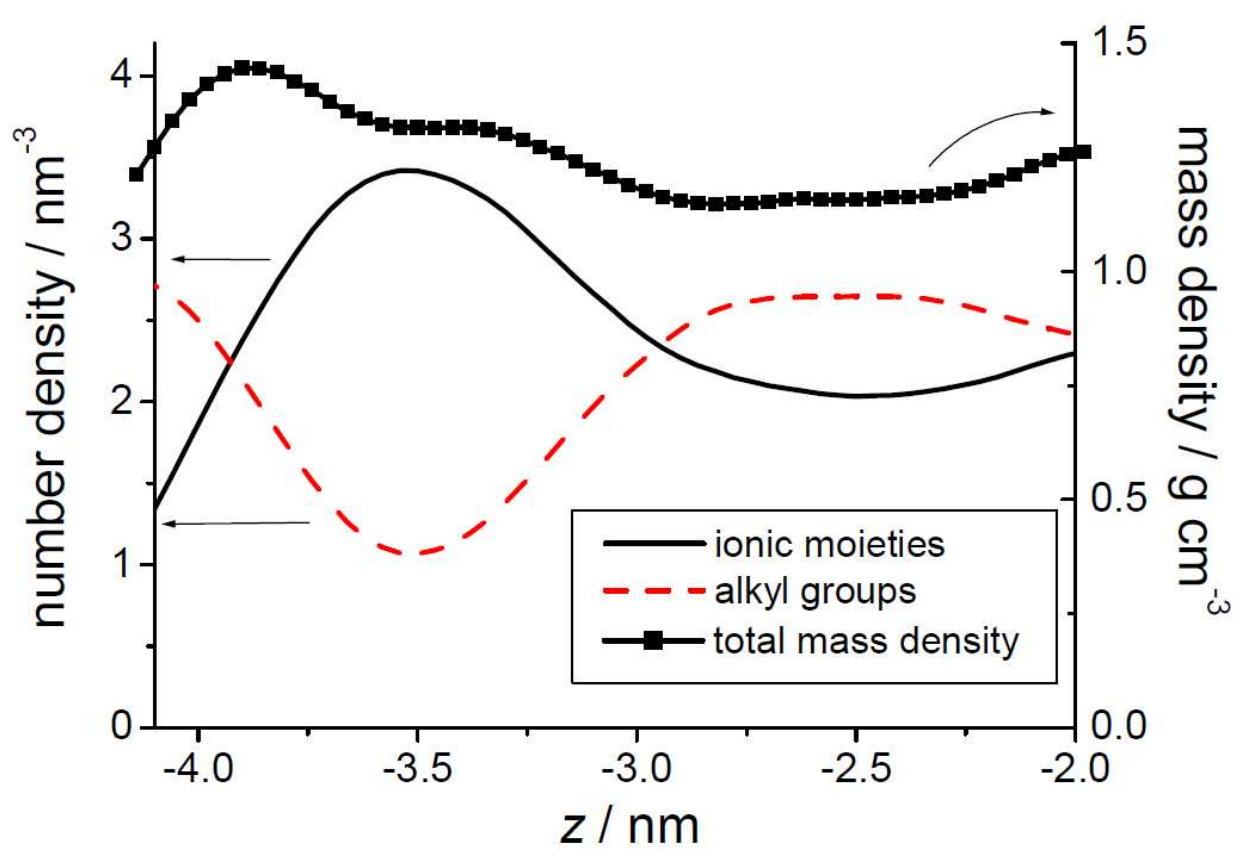

Figure S2 -Global number density profiles averaged over individual sites belonging to ionic moieties (black solid line) and alkyl groups (red dashed line). Global mass density profile for the whole system is also plotted (black squares with solid black line). The scale highlights the region of the density enhancement near the surface, dominated by ionic moieties, and the region of depleted density further into the liquid phase, dominated by alkyl groups. 


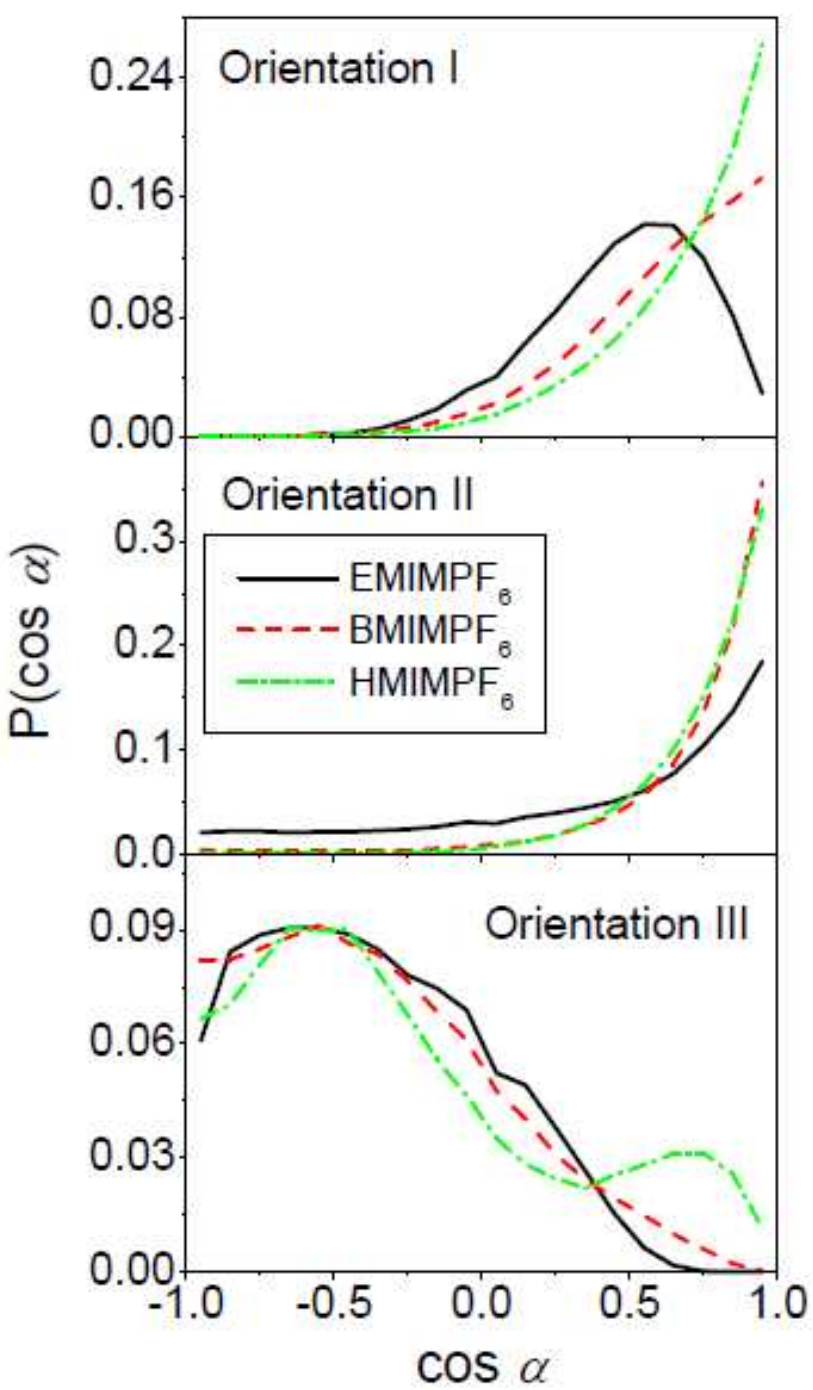

Figure S3 - Probabilities for the angle between the surface normal and the vector pointing from the first carbon atom of the butyl chain (C13) to the terminal one (C16, C22 and C28 for EMIM, BMIM and HMIM, respectively) in ILs with different cation alkyl chain length. The top panel is for rings in orientation I, the middle panel for orientation II and the lower panel for orientation III, as defined by the blue rectangles in Figure 6 of the paper. 


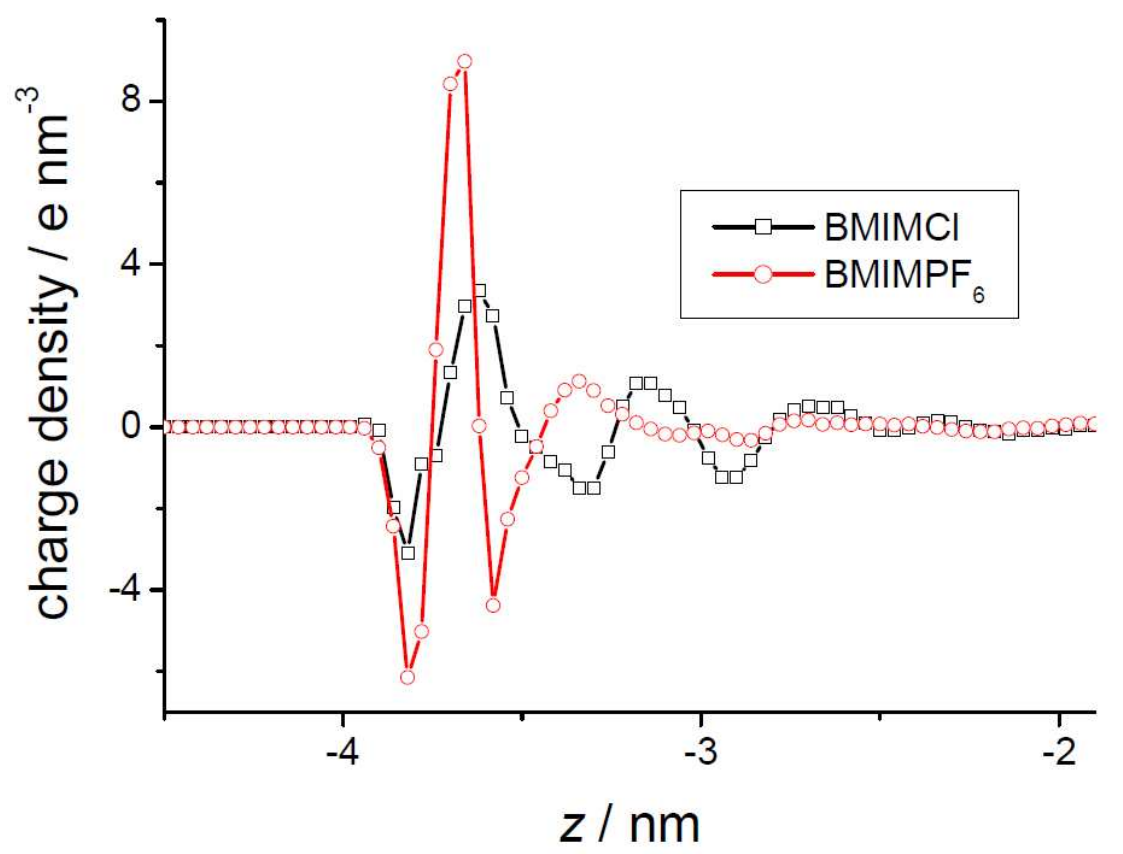

Figure S4 - Intrinsic electron density profiles across the interface of $\mathrm{BMIMCl}$ (open squares, black curve) and $\mathrm{BMIMPF}_{6}$ (open circles, red curve) at $360 \mathrm{~K}$. 


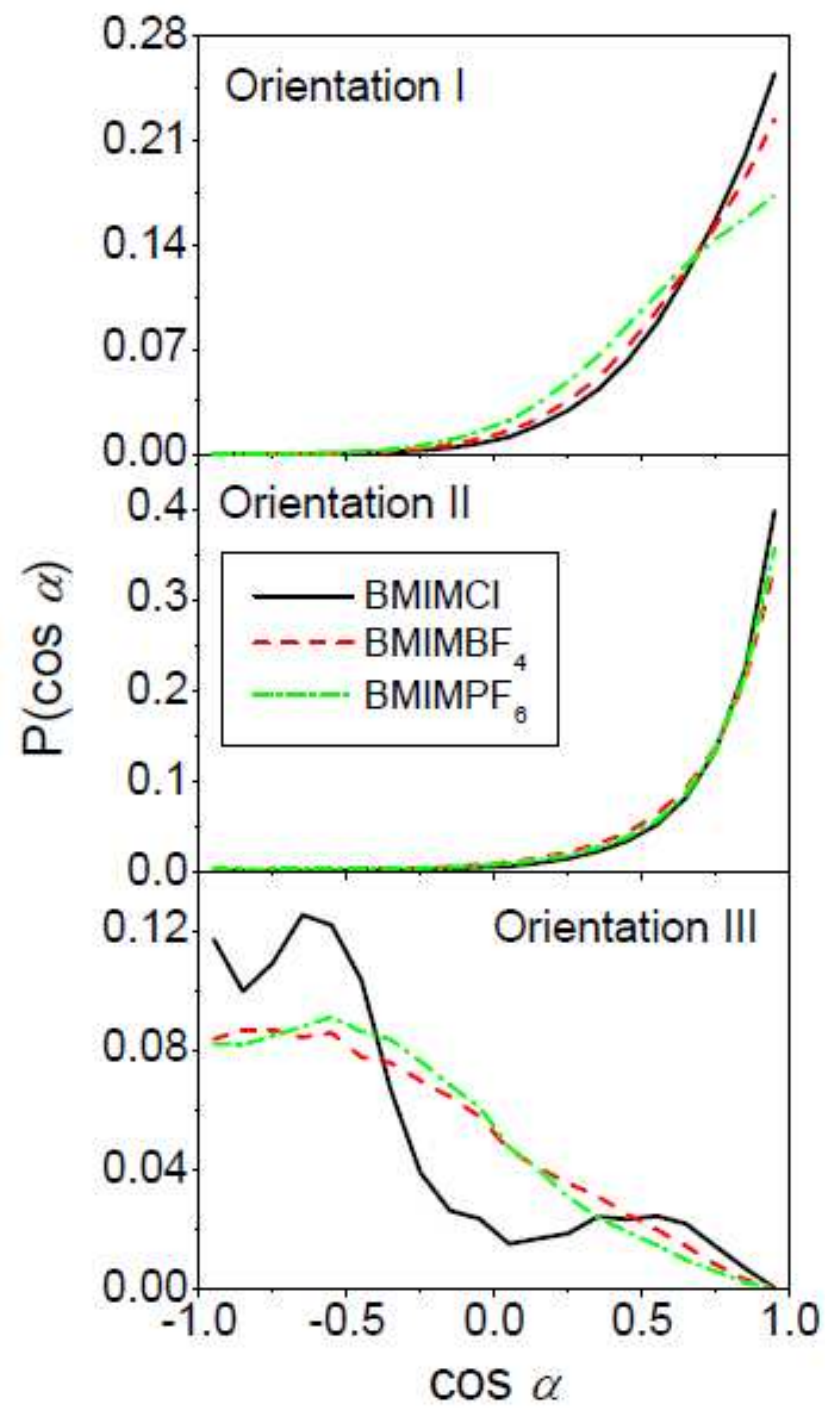

Figure S5 - Probabilities for the angle between the surface normal and the vector pointing from the first carbon atom of the butyl chain (C13) to the terminal one (C22) in ILs with different anions. The top panel is for rings in orientation I, the middle panel for orientation II and the lower panel for orientation III, as defined by the blue rectangles in Figure 6 of the paper. 


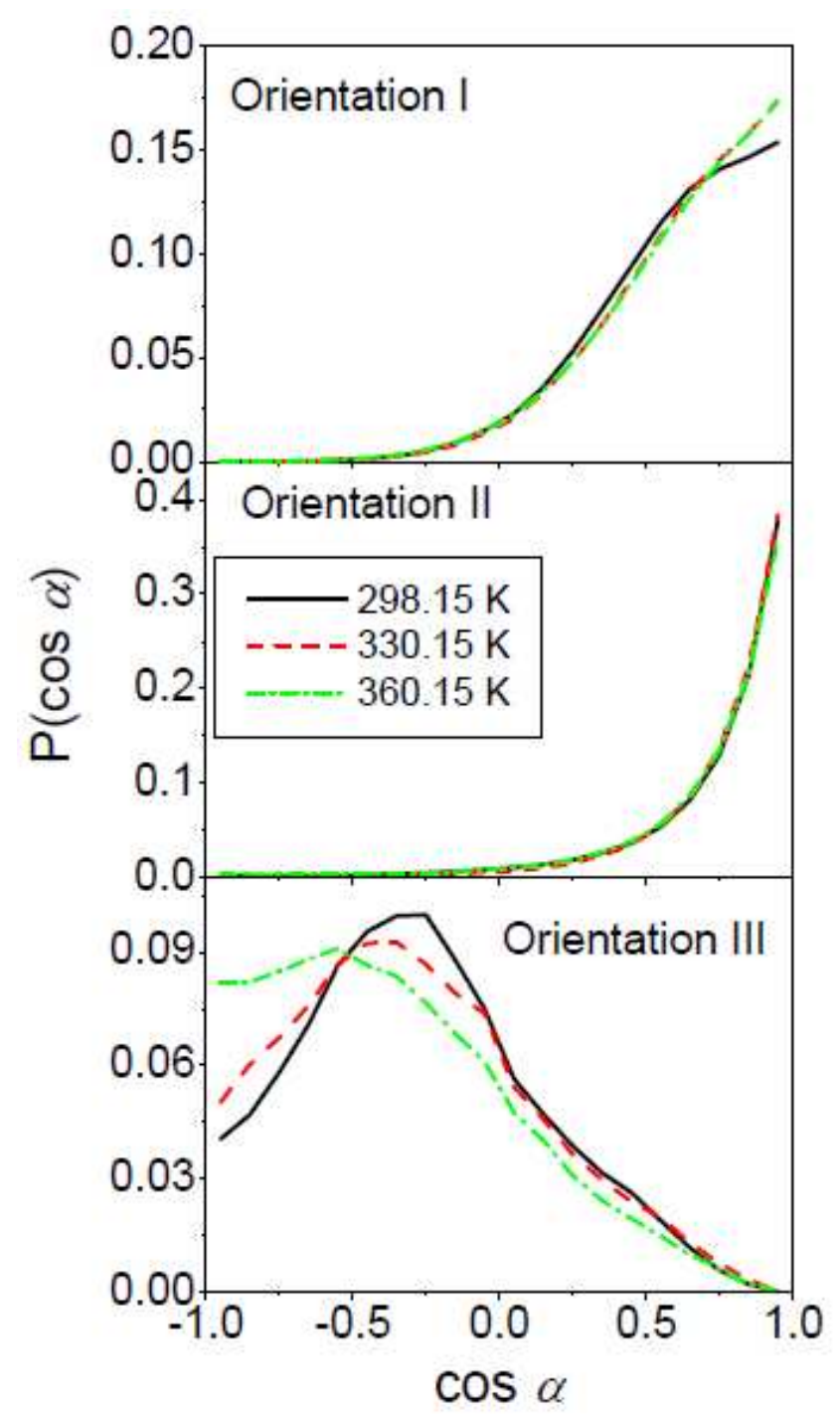

Figure S6 - Probabilities for the angle between the surface normal and the vector pointing from the first carbon atom of the butyl chain (C13) to the terminal one (C22) in $\mathrm{BMIMPF}_{6}$ at different temperatures. The top panel is for rings in orientation I, the middle panel for orientation II and the lower panel for orientation III, as defined by the blue rectangles in Figure 6 of the paper. 


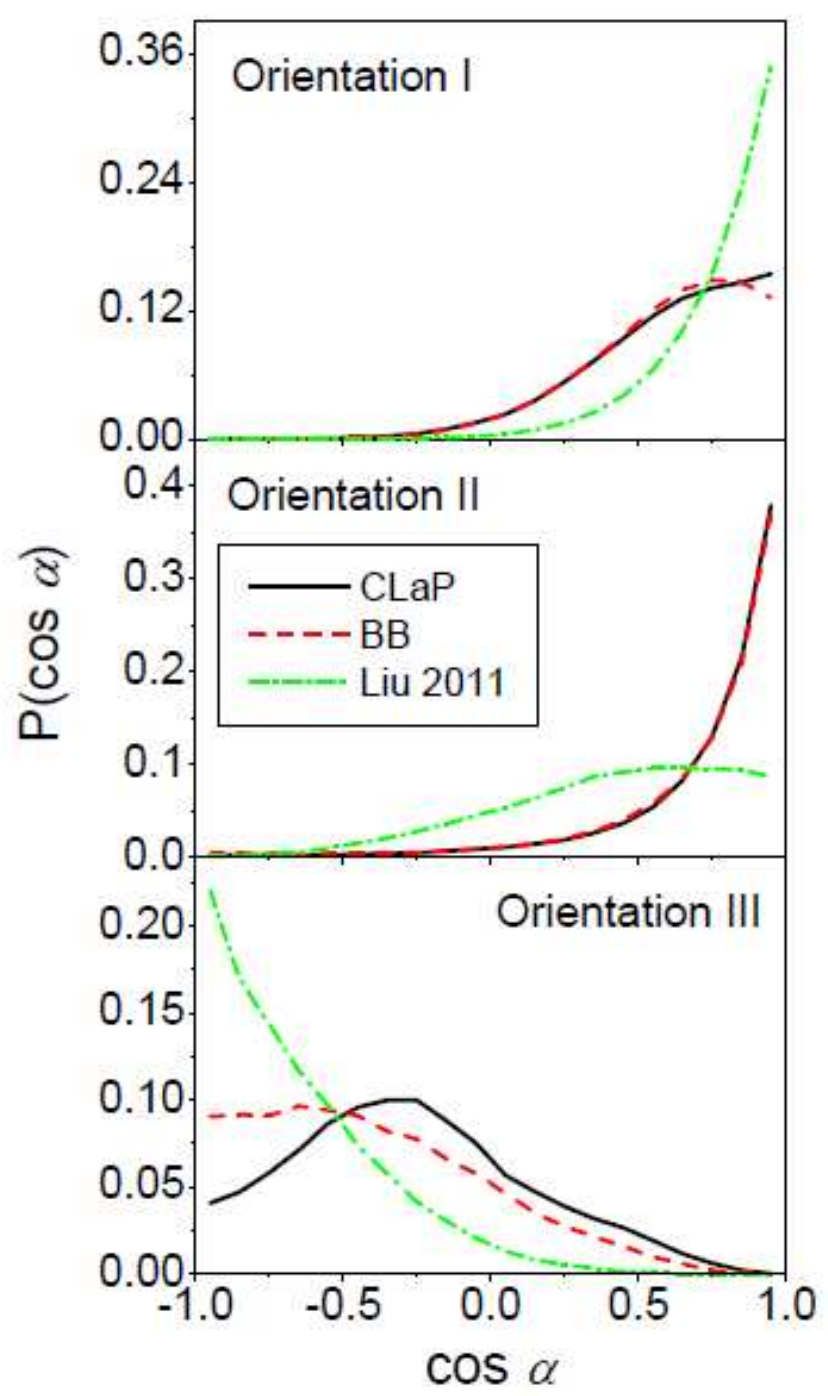

Figure S7 - Probabilities for the angle between the surface normal and the vector pointing from the first carbon atom of the butyl chain (C13) to the terminal one (C22) in $\mathrm{BMIMPF}_{6}$ at $298 \mathrm{~K}$ using different molecular models. The top panel is for rings in orientation I, the middle panel for orientation II and the lower panel for orientation III, as defined by the blue rectangles in Figure 6 of the paper. 